\title{
A Galactosylceramide Binding Domain Is Involved in Trafficking of CLN3 from Golgi to Rafts via Recycling Endosomes
}

\author{
DIXIE-ANN PERSAUD-SAWIN, JAMES O. MCNAMARA II, SVETLANA RYLOVA, \\ ANTONIUS VANDONGEN, AND ROSE-MARY N. BOUSTANY
}

\begin{abstract}
Department of Pediatrics [D.-A.P.-S., S.R., R.-M.N.B.], Department of Neurobiology [J.O.M. II, A.V., R.-M.N.B.], Department of Pharmacology [A.V.], University Program in Genetics and Genomics [D.-A.P.-S, R.-M.N.B.], Duke University Medical Center, Durham, North Carolina, 27710, U.S.A., and Institute of Molecular Biosciences [S.R], Section of Veterinary Biochemistry, Biomedical Center, Uppsala S-75643, Sweden
\end{abstract}

\begin{abstract}
Juvenile neuronal ceroid lipofuscinosis (JNCL) is due to mutations in the CLN3 gene. We previously determined that CLN3 protein harbors a highly conserved motif, VYFAE, necessary for its impact on cell growth and apoptosis. Using molecular modeling we demonstrated that this motif is embedded in a stretch of amino acids that is homologous to and structurally compatible with a galactosylceramide (GalCer) binding domain. This domain is present in the V3 loop of the HIV-1 gp120 envelope protein, $\beta$-amyloid protein, and the infectious form of prionic protein, and defines a binding site for lipid rafts. We determined the subcellular localization of CLN3 in different cell systems including human neurons, primary rat hippocampal neurons, normal human fibroblasts, and JNCL fibroblasts homozygous for the $1.02 \mathrm{~kb}$ deletion in genomic DNA. Wild-type CLN3 protein was present within Golgi, lipid rafts in the plasma membrane, and early recycling endosomes, but not late endosomes/lysosomes. Wild-type CLN3 internalized from the plasma membrane to the Golgi via Rab4- and Rab11-positive recycling endosomes. Wild-type CLN3 co-localized with GalCer in the
\end{abstract}

\section{ABSTRACT}

Golgi and in lipid rafts at the plasma membrane in normal cells. Neither mutant CLN3 protein nor GalCer were found at the plasma membrane in JNCL fibroblasts. Mutant CLN3p was retained within the Golgi and partially mis-localized to lysosomes, failing to reach recycling endosomes, plasma membrane, or lipid rafts. These studies identify a novel CLN3 domain that may dictate localization and function of CLN3. (Pediatr Res 56: 449-463, 2004)
CTH, ceramide trihexoside
Abbreviations
EEA1, early endosomal antigen 1
GalCer, galactosylceramide
GRASP65, Golgi reassembly and stacking protein
JNCL, juvenile neuronal ceroid lipofuscinosis
SM, sphimgomyelin
TGN38, trans Golgi network protein 38
TNF-R1, tumor necrosis factor receptor type 1

JNCL (OMIM 204200) is an autosomal recessive disease caused by mutations in the CLN3 gene. The hallmarks of the disease are blindness due to retinitis pigmentosa, seizures, psychomotor and cognitive decline, and death in the mid- to late $20 \mathrm{~s}$ (1). JNCL is one of the most common childhood neurodegenerative diseases (2). Massive neuronal death in the cerebrum and cerebellum, photoreceptor loss, and the accumu-

Received December 29, 2003; accepted March 31, 2004.

Correspondence: Rose-Mary N. Boustany, M.D., Duke University Medical Center, MSRB Box 2604, Research Dr., Durham, NC 27710, U.S.A.; e-mail: boust001@mc.duke.edu

Supported by National Institute of Neurological Disorders and Stroke grant 5 RO1 NS 43344-2 (R.-M.B)

DOI: 10.1203/01.PDR.0000136152.54638.95 lation of lipids and proteins in lysosomes of surviving pyramidal neurons defines the pathology of $\operatorname{JNCL}(3,4)$.

The precise functions of CLN3, as well as its specific molecular targets, remain to be discovered. Yet, some insights into the pathophysiology have been made. Defects in the CLN3 protein lead to dysregulation of $\mathrm{pH}$ in both yeast and mammalian cells $(5,6)$. Also, ample evidence exists that neuronal death in JNCL is the result of apoptosis (7-13). Inspection of the primary CLN3 sequence led us to identify two short segments, 291VYFAE295 and 330VFASRSSL337, that are highly conserved across species and necessary for the role of the CLN3 protein in regulating cell growth and apoptosis (11). We had established that the CLN3 protein modulates ceramide generation. There is an increase in ceramide levels in CLN3- 
deficient brain and cells. Conversely, CLN3-overexpressing cells generated $70 \%$ less ceramide than did normal cells (12). An increase in ceramide generation was subsequently described in association with another neurodegenerative disease, amyotrophic lateral sclerosis $(14,15)$.

Defining the mechanisms of action of the CLN3 protein, and how this protein impacts apoptosis and ceramide generation, must begin with a clear understanding of its subcellular localization. So far, studies by others have been inconclusive and have placed CLN3 in 1) the mitochondria in mouse retina Müller cells (16), 2) vacuoles in yeast (17), 3) lysosomes in HEK and HeLa cells (18), 4) synaptosomes in PC6-3 neuronal cells $(19), 5)$ the nucleus in rat neurons $(20), 6)$ synapses but not synaptic vesicles (21), and 7) the Golgi and plasma membrane in HeLa, fibroblasts, and COS-6 cells (22).

Mahfoud et al. (23) established that the $\beta$-amyloid precursor protein contains a GalCer binding domain structurally identical to the one in the V3 loop of the HIV-1 surface envelope glycoprotein, gp120, and to that within the infectious form of prionic protein, $\mathrm{PrP}^{\mathrm{sc}}$. This domain consists of a helix-turnhelix structure and two anchoring residues, phenylalanine (F) and tyrosine $(\mathrm{Y})$, that mediate the binding of these proteins to sugar rings of the complex glycosphingolipid, GalCer (24).

We determined that the binding domain described in the $\beta$-amyloid precursor protein contains the motif 19VFFAE22, which is very similar to the stretch of amino acids, 291VYFAE295, within CLN3 that we had previously identified as being necessary for antiapoptotic function. Changing residue $292 \mathrm{Y}$ to $\mathrm{F}$ within CLN3 had no effect on the impact of this protein on cell growth and apoptosis (11). Analyzing published mutations in Alzheimer's and prionic diseases led us to the following interesting observations: the known human CLN3 mutation E295K (25) and analogous mutations within the $\operatorname{PrP}^{\mathrm{sc}}$ (E220K) and $\beta$-amyloid (E22K) proteins all result in apoptosis and disease $(26,27)$. This structural domain allows these proteins to bind to GalCer and to insert into lipid rafts and/or caveoli in the plasma membrane (PM). These liquid-ordered microdomains function as platforms for receptor binding and initiation of signaling cascades, and they are essential for orchestrating these processes in a spatially organized manner. These microdomains consist primarily of sphingolipids and cholesterol, are derived from the Golgi, and are involved in protein trafficking and endocytotic pathways $(27,28)$. Proteins containing two lipid acyl chains (myristoyl and palmitoyl) associate with caveolae, and those with a prenyl tail bind to lipid rafts (29). A potential myristoylation site is present within CLN3, but actual myristoylation of the protein has never been proven (30). The CLN3 protein, however, was shown to be prenylated $(31,32)$.

In this study, the cellular geography and movement of the unmodified wild-type and naturally occurring mutant CLN3 proteins are described: CLN3 protein was present in lipid rafts at the PM, in the Golgi and early recycling endosomes, and probably traffics between the Golgi and PM via early recycling endosomes. Molecular modeling of the putative GalCer binding domain in CLN3, which includes the 291VYFAE295 motif, the aromatic anchoring residues Y284 and F287 outside this motif, as well as surrounding residues inclusive of amino acids 274-302 produced an alignment structurally compatible with the GalCer/lipid raft binding domain common to $\beta$-amyloid, $\operatorname{PrP}^{\mathrm{sc}}$, and the V3 loop of the gp120 protein. In support of the CLN3/GalCer binding model, wild-type CLN3p colocalized with GalCer in lipid rafts and in the Golgi. Mutant CLN3 protein lacking the GalCer binding domain was primarily retained within a fragmented Golgi, mis-localized to late endosomes/lysosomes, and failed to reach early recycling endosomes and lipid rafts.

\section{METHODS}

\section{Cell Lines}

Human neuronal precursor cells (hNT2) (Stratagene, La Jolla, CA, U.S.A.); primary rat hippocampal neurons (BrainBits, SIU Medical School, Chicago, IL, U.S.A.); normal human fibroblasts and CLN3-deficient fibroblasts homozygous for the $1.02 \mathrm{~kb}$ deletion in genomic DNA (Duke University, Durham, NC, U.S.A.) were used for subcellular studies. The same patient cell line was used in all experiments. Use of patient cells is covered by a Duke University Institutional Review Board-approved protocol.

\section{Tissue Culture}

Fibroblasts and hNT2 neurons were grown in high-glucose Dulbecco's modified Eagle's medium with $10 \%$ fetal bovine serum (FBS), 1\% antibiotic (Invitrogen, Carlsbad, CA, USA) at $37^{\circ} \mathrm{C}$ and $5 \% \mathrm{CO}_{2}$. Primary rat hippocampal neurons were grown in neurobasal/glutamine medium (Invitrogen). Cells were grown overnight on poly-D-lysine-treated coverslips at a density of $1-5 \times 10^{4}$ cells $/ 0.44 \mathrm{~cm}^{2}$.

Production of postmitotic human neurons. hNT2 precursor cells were plated in T75 flasks at a density of $2 \times 10^{6} /$ flask and allowed to attach. Cells were treated with $10 \mu \mathrm{M}$ retinoic acid twice per week for $4 \mathrm{wk}$. Retinoic acid was added to the media at 1:1. Following this, cells were replated at lower densities, 1:4 or 1:6 and allowed to sit for $2 \mathrm{~d}$. The cells were then trypsinized $(1 \times$ trypsin for $30 \mathrm{~s})$, counted and replated at $1 \times$ $10^{5}$ per well in 24 -well plates. The media was saved as "conditioned media" for future replates. The cells were then treated with $1 \mu \mathrm{M}$ cytosine arabinoside (Sigma Chemical Co., St. Louis, MO, U.S.A.), $10 \mu \mathrm{M}$ fluorodeoxyuridine, and 10 $\mu \mathrm{M}$ uridine two to three times per week for $2 \mathrm{wk}$. The postmitotic neurons were harvested and then frozen in $95 \%$ $\mathrm{FBS} / 5 \%$ DMSO until use.

\section{pEYFP-CLN3 Construction}

The yellow fluorescent protein (YFP) gene was cloned in frame into the pGEMCLN3 vector between amino acid residues 250 and 251, in the second external loop $(11,30)$. Sequence was verified by the Duke University Sequencing Facility. The site for insertion of the YFP gene was chosen to avoid potential targeting signals at the amino and carboxyl termini. CLN3 mutant constructs lacking exons 8-15 from the carboxyl end of the cDNA (CLN3 $\Delta 8-15$ ) or coding for a protein missing the amino acids VYFAE (CLN3 $\triangle$ VYFAE) 
were generated by site-directed mutagenesis before tagging with the YFP gene (11).

\section{Transfection}

Fibroblasts were plated on coverslips $1 \mathrm{~d}$ before transfection and neurons plated 4-6 d before transfection. Only fibroblasts and primary rat hippocampal neurons were transfected with localization vectors using the Lipofectamine 2000 per the manufacturer's protocol (Invitrogen). Transfection efficiency was determined using $\beta$-gal chemiluminescent detection kit (CLONTECH, Palo Alto, CA, U.S.A.), and was determined to be between 60 and $70 \%$ for fibroblasts and 80 and $90 \%$ for primary rat neurons.

\section{Immunocytochemistry}

Controls. For each experiment, the following controls were used: 1) all components excluding one or both primary antibodies and 2) all components excluding both secondary antibodies. In all instances, exclusion of the primary antibody resulted in no signal intensity for the particular protein, including CLN3p. Exclusion of the secondary fluorescent antibodies also resulted in low-to-no signal intensity in the respective fluorescent channels. For transfection experiments, untransfected cells were used as a negative control in addition to the above controls.

CLN3 visualization. The polyclonal anti-CLN3 antibody was raised against residues $58-77$ of the CLN3p in rabbits (11, $33)$. This antibody has been extensively characterized. Since it has been raised to the amino terminal of the CLN3 protein it also detects the mutant CLN3 protein with a $1.02-\mathrm{kb}$ deletion in the genomic DNA, in addition to numerous other deletions and point mutations (see Fig. 7 in ref. 11). This antibody has also been used semiquantitatively in immunohistochemical studies (see Figs. 3 and 5 in ref. 33). On Western blots, it detects a doublet band at $48-52 \mathrm{kD}$. This antibody has also been used successfully in co-immunoprecipitation experiments (unpublished). pEYFP-CLN3, pEYFP-CLN3 $\Delta 8-15$, and pEYFP-CLN3 $\triangle$ VYFAE were also used for detection of normal and mutant CLN3 protein distribution patterns.

Staining protocol. Cells were washed with Dulbecco's PBS (DPBS) at room temperature and fixed with ice-cold fixative solution ( $4 \%$ paraformaldehyde $/ 4 \%$ sucrose $/ 1 \times$ DPBS) at $4{ }^{\circ} \mathrm{C}$ for $20 \mathrm{~min}$. The cells were washed with ice-cold DPBS and permeabilized with methanol at $-20^{\circ} \mathrm{C}$ for $20 \mathrm{~min}$. This was followed by two washes with blocking solution $(10 \%$ normal goat serum $/ 2 \% \mathrm{BSA} / 1 \times \mathrm{DPBS}$ ) and incubation in block for $1 \mathrm{~h}$ at room temperature. Cells were incubated with a 1:1000 dilution of the CLN3 antibody in block along with a 1:100 1:1000 dilution (in block) of the primary antibody for each organellar protein of interest for $1 \mathrm{~h}$ at room temperature. This was followed by two 10-min washes. Cells were incubated for $1 \mathrm{~h}$ at room temperature in a dilution (1:1000) of the appropriate fluorescent secondary antibodies in block (Molecular Probes, Eugene, OR, U.S.A.). Cells were then washed in block and then three times with DPBS, mounted onto a glass slide with $50 \mu \mathrm{L}$ of fluoromount mounting media (Southern Biotechnology Associates, Birmingham, AL, U.S.A.) and viewed with a Zeiss Laser Scanning Confocal Microscope (LSM510; Carl Zeiss Inc., Thornwood, NY, U.S.A.) at a magnification of $100 \times$. Detection of CLN3p localization in overexpression systems was also determined using transfected normal and CLN3-deficient human fibroblasts and rat neurons. Where possible, cells were transfected with the vector containing the organelle marker protein, washed, fixed and permeabilized as described above, and incubated with the CLN3 antibody as previously described.

Golgi visualization. Localization was carried out using the rat monoclonal anti-GRASP65 (Dr. Francis Barr, Max Planck Institute, Martinsried, Germany), and antibodies to TGN38 (Santa Cruz Biotechnology, Santa Cruz, CA, U.S.A.) and anti-TNF-R1 (Research Diagnostics, Flanders, NJ, U.S.A.). Cells were also transfected with pECFP-Golgi vector which codes for $\beta$-1,4galactosyl transferase (CLONTECH).

Mitochondria visualization. Studies were carried out using MitoTracker Green (Molecular Probes). Cells were also transfected with the pECFP-mitovector that codes for cytochrome oxidase (CLONTECH).

Endoplasmic reticulum (ER) visualization. Studies were carried out using an antibody to calreticulin (Research Diagnostics). Cells were also transfected with the pECFP-ER vector that codes for calreticulin (BD Biosciences, CLONTECH).

Nucleus visualization. Studies were carried out using a cocktail of antibodies to nuclear lamins (Research Diagnostics), or stained with propidium iodide as previously described (11). Cells were also transfected with pECFP-Nuc vector (NLS of SV40) (CLONTECH).

Endosome/lysosome visualization. Studies were carried out using antibodies to cathepsin D (Santa Cruz Biotechnology), LAMP1 (Research Diagnostics), Rab4 and Rab11 (CLONTECH), and EEA1 (Dr. Mike Ehler, Duke University). Cells were also transfected with pECFP-Endo (RhoB), pDSRED vectors containing Rab4, Rab5, Rab7, and Rab11 proteins (The Guthrie Institute, Sayre, PA, U.S.A.) and YFPTGN38.

Speed of internalization of CLN3 and Rab4 and Rab11 proteins. For determination of the velocity of internalization of the CLN3 protein, cells were washed in $37^{\circ} \mathrm{C}$ DPBS, and then incubated with the CLN3 antibody and either Rab4 or Rab11 antibody in $37^{\circ} \mathrm{C}$ DPBS for varying times of $2,5,10$, and 15 min at $37^{\circ} \mathrm{C}$. The cells were immediately placed on ice and taken into a cold room maintained at $4^{\circ} \mathrm{C}$. They were washed three times with ice-cold DPBS, then exchanged with ice-cold acid wash $(0.2 \mathrm{M}$ acetic acid/ $0.5 \mathrm{M} \mathrm{NaCl})$, and incubated for 5 min, and then washed three times with ice-cold DPBS. Cells were fixed with ice-cold fixative solution (4\% paraformaldehyde $/ 4 \%$ sucrose $/ 1 \times$ DPBS) at $4^{\circ} \mathrm{C}$ for $20 \mathrm{~min}$. This was followed by three washes with DPBS and permeabilization with methanol at $-20^{\circ} \mathrm{C}$ for $20 \mathrm{~min}$. The methanol was discarded and DPBS (room temperature) added immediately. The cells were washed with block (10\% normal goat serum/ $2 \% \mathrm{BSA} / 1 \times \mathrm{DPBS}$ ) at room temperature for $1 \mathrm{~h}$ and then incubated with the secondary antibody for $1 \mathrm{~h}$ at room temperature. The cells were washed in block three times, once with DPBS, and then viewed as described above. Two labeled sister cultures at $4{ }^{\circ} \mathrm{C}$, with and without acid washing, were included as controls. 
Plasma membrane and lipid raft visualization. Studies were carried out using antibodies to TNF-R1, Gap43 (neuromodulin), alkaline phosphatase, and caveolin 1 (Research Diagnostics). Cells were also transfected with the pECFP-Mem (Gap43) vector (CLONTECH). Surface labeling of cells with CLN3 was also carried out.

Surface labeling. Cells plated for surface labeling were placed at $4^{\circ} \mathrm{C}$ for $30-60 \mathrm{~min}$. Living cells were washed three times in ice-cold DPBS in the cold room. The CLN3 antibody was then added (1:1000 dilution/DPBS) and incubated at $4{ }^{\circ} \mathrm{C}$ for $30 \mathrm{~min}$. The cells were washed three times in ice-cold DPBS and fixed with ice-cold fixative solution (4\% paraformaldehyde $/ 4 \%$ sucrose $/ 1 \times$ DPBS) at $4{ }^{\circ} \mathrm{C}$ for $20 \mathrm{~min}$ followed by one wash with DPBS. The cells were incubated with the secondary antibody (1:500 dilution/block) for $1 \mathrm{~h}$ at room temperature. Cells were washed in block three times, and then once with DPBS before viewing.

BODIPY-GalCer labeling. BODIPY-GalCer analog (\#D7519, Molecular Probes) was reconstituted according to the manufacturer's protocol in defatted BSA (Sigma Chemical Co.). Cells were incubated at $4^{\circ} \mathrm{C}$ for $30 \mathrm{~min}$ in $2.5 \mu \mathrm{M}$ GalCer/BSA, washed in $10 \mathrm{mM}$ HMEM (Invitrogen) and incubated for $30 \mathrm{~min}$ to $1 \mathrm{~h}$ at $37^{\circ} \mathrm{C}$. Nonpermeabilized cells were fixed in $3.2 \%$ PFA/PBS for $30 \mathrm{~min}$ at room temperature, quenched with $2.5 \mathrm{mM}$ glycine/PBS for $15 \mathrm{~min}$ at room temperature, and incubated in block $(10 \%$ horse serum in PBS, Invitrogen) for $1 \mathrm{~h}$ at room temperature. Cells were then incubated with CLN3 antibody, followed by the secondary as described previously. For permeabilized cells only, six "backexchange" washes in 5\% defatted BSA/HEPES-buffered minimum Eagle's medium for $10 \mathrm{~min}$ each before fixing (as above). Cells were permeabilized by treating with $0.05 \%$ Trition $\times 100$ for $2 \mathrm{~min}$ after the quenching stage. In addition to the controls listed above, the following were included: i) for nonpermeabilized cells, "back-exchange" washes in defatted BSA were carried out, and ii) for permeabilized cells, a sister culture was labeled and "back exchange washing" was omitted.

\section{Time-Lapse Microscopy}

Primary rat hippocampal neurons were cultured in MatTek dishes (Ashland, MA, U.S.A.) at a density of $1.5 \times 10^{4}$ cells per dish, and transfected with the pEYFP-CLN3 vector previously described. Live cell epi-fluorescence images were captured using a $40 \times$ oil objective, YFP filter sets (Chroma Technology Corp., Rockingham, VT, U.S.A.) and a 12-bit cooled CCD camera (ORCA, Hamamatsu Corp., Bridgewater, NJ, U.S.A.) on an inverted microscope (Diaphot, Nikon, Melville, NY, U.S.A.). Image acquisition was controlled by Openlab software (Improvision Inc., Lexington, MA, U.S.A.). Series of images were taken in 50 frames of $1 \mathrm{~s}$ exposure with a 0.4-s delay between frames. The frames were acquired $1.4 \mathrm{~s}$ apart with a total running time of $70 \mathrm{~s}$. Video can be viewed at www.dbsr.duke.edu/pub/cln3.

\section{Protein Modeling}

Amino acid residues 274-302 of the CLN3 protein sequence (GI: 4502888 ) were modeled onto residues $1-28$ of the $\beta$-amy- loid precursor protein using the Protein Data Bank model 1BJB. The secondary-structure preferences, structural alignment, and fit of rotameric side-chain conformations were assessed using the SWISSPROT PDB viewer and the MAGE/ PROBE molecular graphics program (available at: http:// kinemage.biochem.duke.edu) (34).

\section{${ }^{14}$ C-Palmitate Labeling}

Fibroblasts from a normal control and a CLN3-deficient patient were used in this experiment. For $3 \mathrm{~d}, 1 \times 10^{7}$ cells were grown and labeled with $1 \mu \mathrm{Ci} / \mathrm{mL}{ }^{14} \mathrm{C}$-palmitate. The cells were pelleted and the radioactivity then measured. The washed cell pellet was dissolved in $1 \mathrm{~mL}$ cold distilled water and sonicated. A $100-\mu \mathrm{L}$ aliquot was used for protein determination and incorporated radioactivity. The rest was added to $2 \mathrm{~mL}$ methanol.

Lipid Extraction. Twenty-five micrograms of neutral glycosphingolipid Qualmix (Matreya, Inc., Pleasant Gap, PA, U.S.A.), $15 \mu \mathrm{g}$ GalCer, $20 \mu \mathrm{g}$ GluCer, $2 \mathrm{~mL}$ chloroform, and $1.1 \mathrm{~mL}$ water were added to the samples. The lower organic phase was dried under nitrogen. The upper phase was washed with chloroform and dried. Total radioactivity incorporated into lipids was obtained by dissolving the dry lipids in $500 \mu \mathrm{L}$ of a 1:1 chloroform:methanol mix. Aliquots of $5 \mu \mathrm{L}$ in $5 \mathrm{~mL}$ of Econofluor (Perkin-Elmer, Boston, MA, U.S.A.) were counted and counts multiplied by 100 .

Methanolysis. Total lipids were dissolved in $0.5 \mathrm{~mL}$ chloroform:methanol mix, and $0.25 \mathrm{~mL}$ chloroform, $0.15 \mathrm{~mL}$ methanol, and $0.1 \mathrm{~mL} \mathrm{NaOH}$ in methanol added, and incubated at $37^{\circ} \mathrm{C}$ for $1 \mathrm{~h} ; 0.1 \mathrm{~mL} 2 \mathrm{~N}$ HCL was added for $10 \mathrm{~min}$. The samples were run three times in ether, once in a $65-38-8$ mix (chloroform:methanol:2.5 $\mathrm{M}$ ammonium hydroxide) and exposed to iodine vapor. The band positions were marked.

Thin layer chromatography (TLC). The lower phase of lipid extract was dried and dissolved in $50 \mu \mathrm{L}$ of a 2:1 mix of chloroform:methanol. Thirty microliters was spotted onto a TLC plate and sprayed with $1 \%$ borax decahydrate and dried, then activated at $110^{\circ} \mathrm{C}$ for $1.5 \mathrm{~h}$, cooled down, dried, and exposed to a low-energy phosphoimager screen for $7 \mathrm{~d}$. The bands were scraped, counted, and expressed as $\mathrm{cpm} / \mu \mathrm{g}$ of protein.

\section{Measurement of Ceramide}

$1 \times 10^{6}$ cells each of CLN3-deficient and normal fibroblasts were transfected with intact CLN3 or an empty vector and harvested. Ceramide levels were determined by mass spectrometry at the Lipidomics Core at the Medical University of South Carolina.

\section{RESULTS}

Subcellular localization of the wild-type CLN3 protein. The subcellular localization of unmodified, wild-type CLN3 protein was determined in human and rat neurons, as well as human fibroblasts, using dual-immunolabeling with antibodies for CLN3 and a number of known organellar proteins, and subsequent visualization by laser scanning confocal microscopy. In 
several instances, findings were corroborated using fluorescently labeled YFP-CLN3 and co-localizing vectors and/or antibodies. In the cell systems used, we found that unmodified CLN3 protein and fluorescently labeled YFP-CLN3 were distributed similarly in primary rat hippocampal neurons and normal human fibroblasts. Insertion of the yellow fluorescent protein (YFP) into the second external loop of the CLN3 protein and use of this construct in co-localization studies produced a pattern similar to that obtained with the CLN3 antibody, demonstrating that the introduction of a bulky protein into the CLN3 protein did not affect its subcellular localization. Insertion of the YFP protein at the amino or carboxyl termini was not used to avoid interfering with targeting signals. Human postmitotic hNT neurons are not readily transfectable. In this cell system, only dual antibody labeling was used for co-localization.

Overall, CLN3 protein resided in Golgi, recycling endosomes, and plasma membrane (PM). A pertinent negative result was the absence of CLN3 protein from lysosomes in human fibroblasts, hNT2 neurons, or rat hippocampal neurons. This was confirmed by lack of co-localization with the lysosomal enzyme, cathepsin D (Fig. 1A), LAMP1 (lysosomal associated membrane protein) (Fig. 1, $C$ and $E$ ), or with the late endosome/lysosome-specific marker, Rab7 (Fig. $1 G)$. These results negate the presence of wild-type CLN3 in late endosomes/lysosomes. The presence of CLN3 protein in Golgi was strongly supported by results from dual-labeling studies with antibodies to CLN3 protein and the Golgispecific markers GRASP65 (Golgi reassembly and stacking protein), and TGN38 (a trans Golgi network protein that is also found in recycling endosomes). CLN3 co-localized with GRASP65 in normal fibroblasts (Fig. $2 A$ ) and, with TGN38 in rat neurons (Fig. 2E), hNT2 neurons (Fig. $2 F$ ), and normal fibroblasts (Fig. $2 G$ ). Co-localization of CLN3 protein with $\beta$-1,4-galactosyl transferase $(\beta-1,4-$ GalT), an enzyme that transfers a galactose to proteins or the glyco-
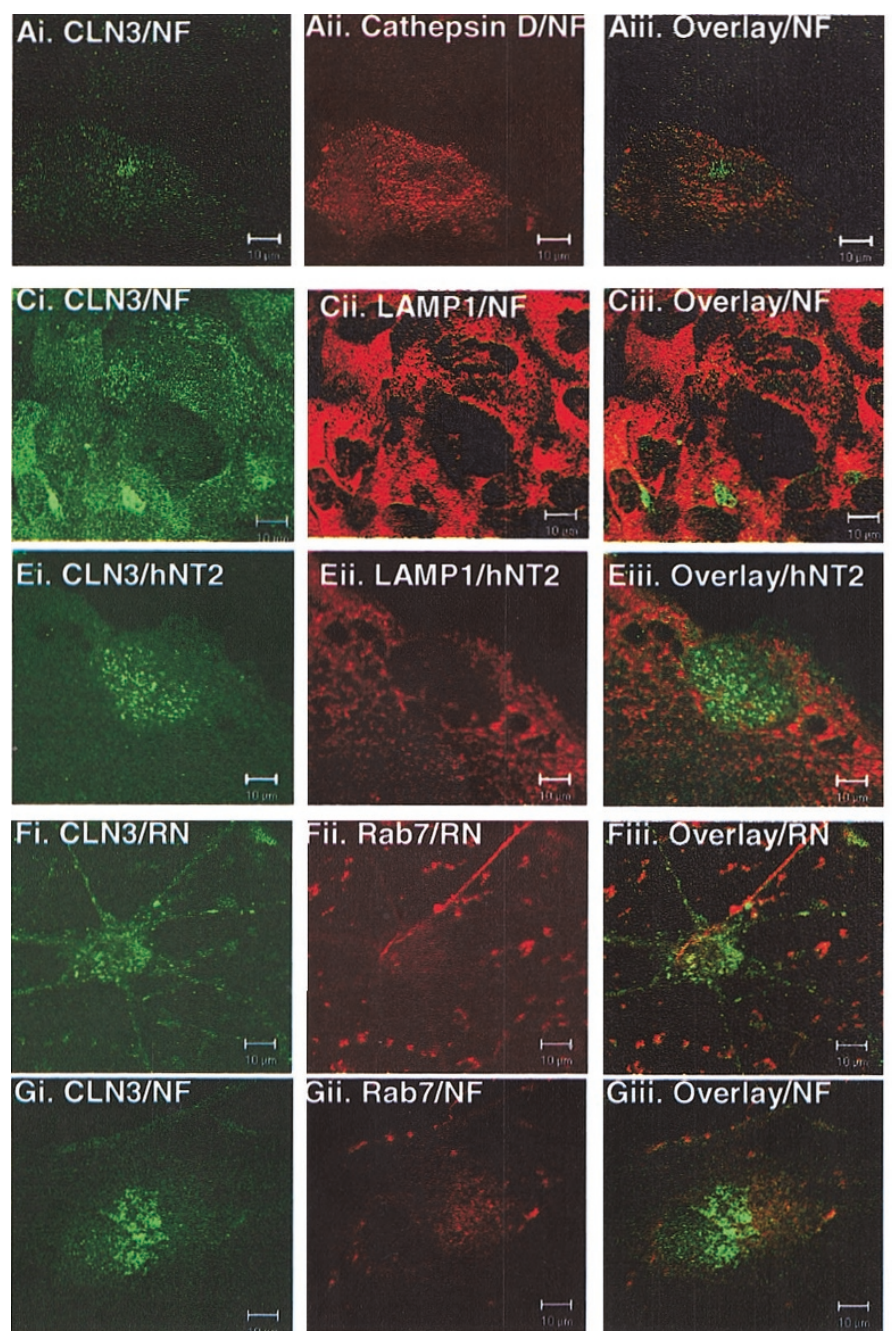
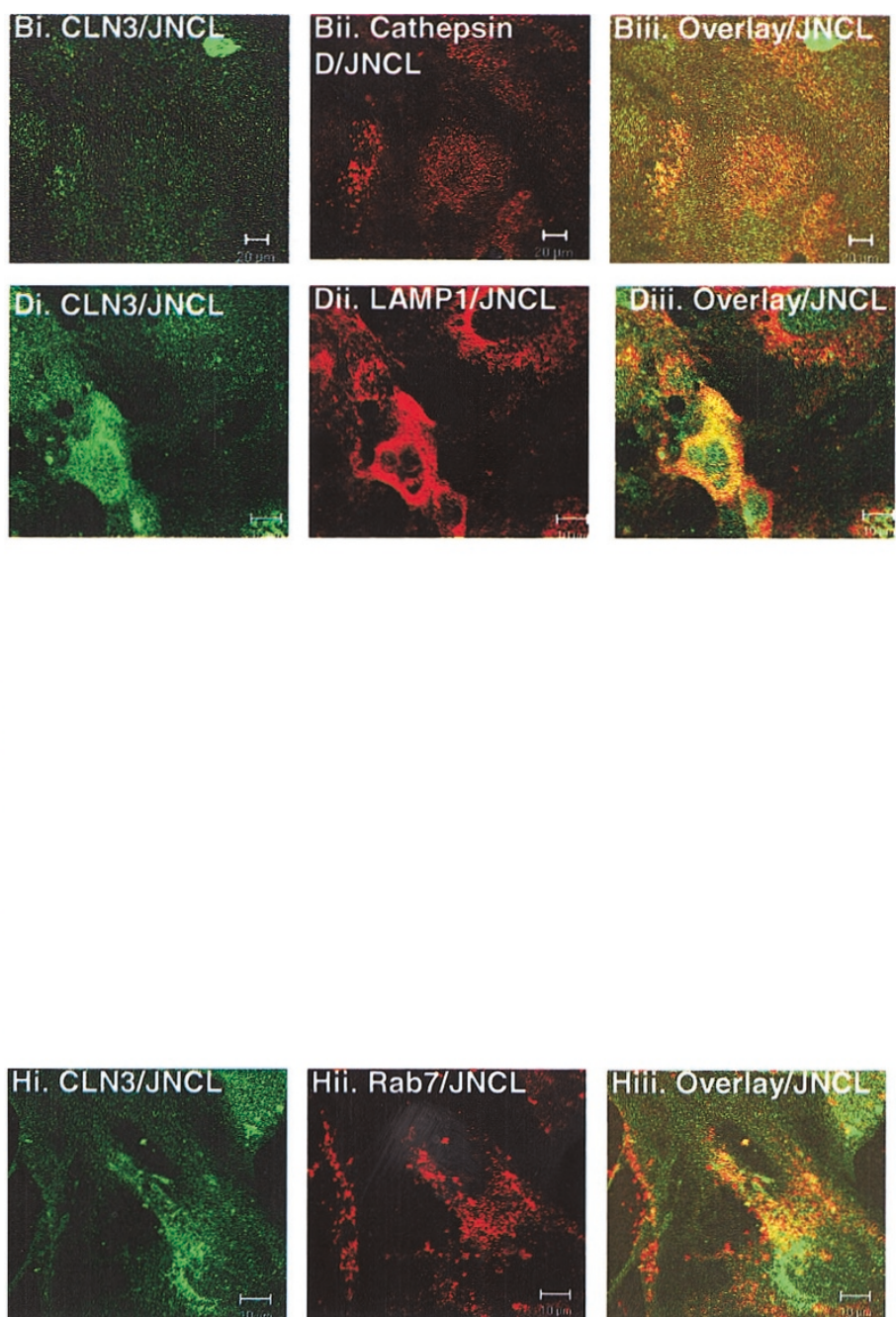

Figure 1. CLN3 and endosomes/lysosomes. (A) Immuno-localization of wild-type CLN3p and cathepsin D in normal fibroblasts. (i) CLN3 is green. (ii) Cathepsin D is red. (iii) Overlay. Regions of co-localization are yellow. (B) Co-localization of mutant CLN3p and cathepsin D to lysosomes in JNCL patient fibroblasts. (i) CLN3 is green. (ii) Cathepsin D is red. (iii) Overlay. Regions of co-localization are yellow. (C) Immuno-localization of wild-type CLN3p and LAMP1 in normal human fibroblasts. $(D)$ Co-localization of mutant CLN3p and LAMP1 to late endosomes/lysosomes in JNCL fibroblasts. (E) Immunolocalization of wild-type CLN3p and LAMP1 in normal human neurons. (i) CLN3 is green. (ii) LAMP1 is red. (iii) Overlay. Regions of co-localization are yellow. Immuno-localization of CLN3p and Rab7p are shown in primary rat hippocampal neurons $(F)$, normal human fibroblasts $(G)$, and CLN3-deficient cells (H). (i) CLN3 protein is green. (ii) Rab7 protein is red. (iii) Overlay. Regions of co-localization are yellow. Cells viewed at a magnification of $100 \times$. $N F$, normal fibroblasts; JNCL, JNCL fibroblasts; $h N T 2$, human neurons; $R N$, rat hippocampal neurons. 

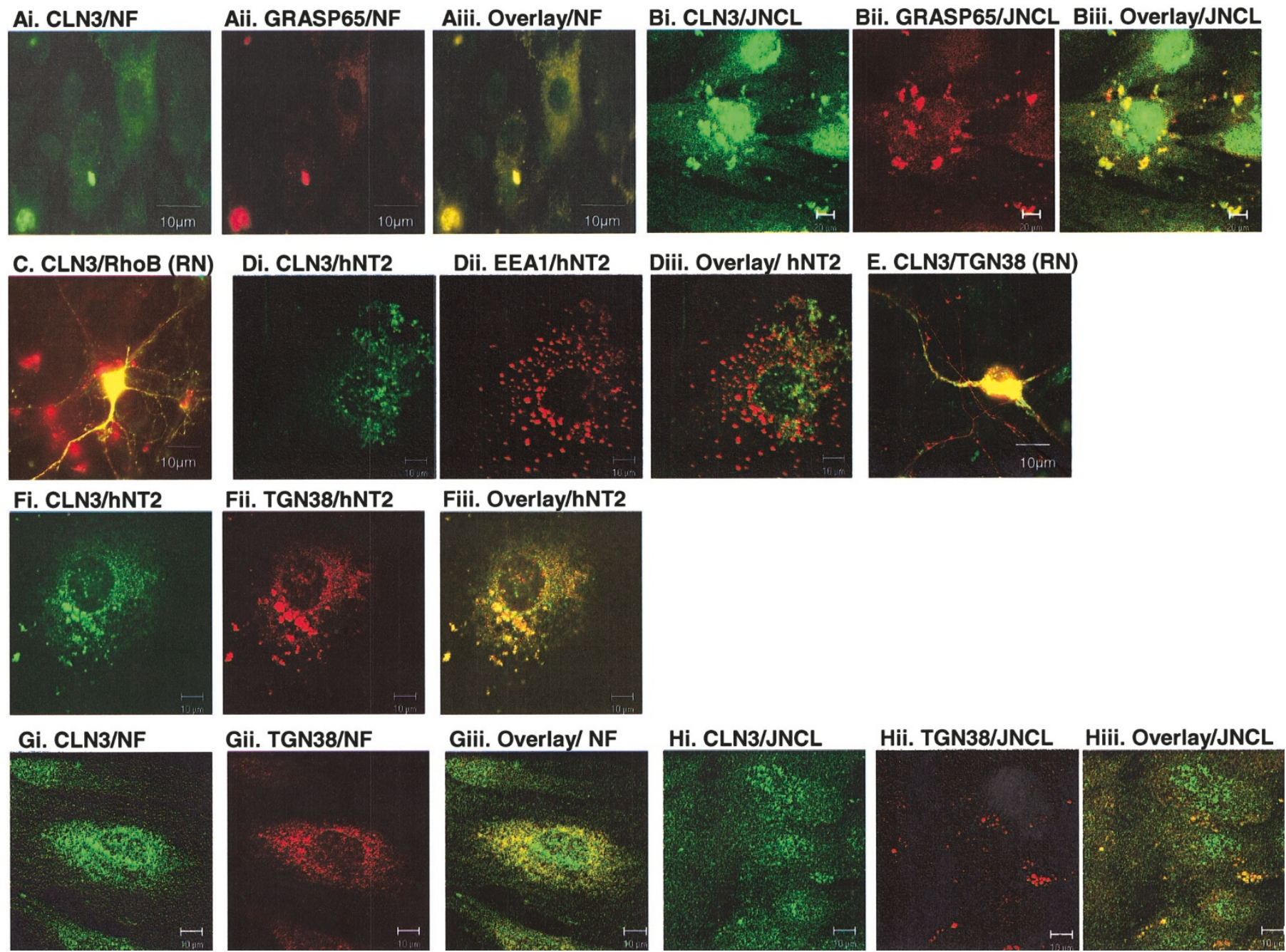

Figure 2. CLN3 protein, Golgi, and early recycling endosomes. CLN3 and GRASP65 proteins are visualized in normal $(A)$ and JNCL $(B)$ fibroblasts. $(i)$ CLN3 is green. (ii) GRASP65 is red. (iii) Overlay. Regions of co-localization are yellow. (C) Overlay of primary rat hippocampus neurons double-labeled with CLN3 (green) and RhoB (red). (D) Human hNT2 neurons dually labeled with CLN3 and EEA1 antibodies. (i) CLN3 is green. (ii) EEA1 is red. (iii) Overlay. (E) Co-localization of CLN3p (green) and TGN38 (red) in rat hippocampal neurons. Regions of co-localization are yellow. Co-localization of CLN3 and TGN38 proteins is shown in human hNT2 neurons $(F)$, normal $(G)$, and CLN3-deficient human fibroblasts $(H)$. (i) CLN3 is green. (ii) TGN38 is red. (iii) Overlay. Regions of co-localization are yellow. Cells were viewed at a magnification of $100 \times . N F$, normal fibroblasts; JNCL, JNCL fibroblasts; $h N T 2$, human neurons; $R N$, rat hippocampal neurons.

sphingolipid glucosylceramide, was also established (results not shown).

CLN3 co-localized with the RhoB protein in rat neurons (Fig. 2C). This placed CLN3 in a broad endosomal pool that encompasses early sorting endosomes and recycling endosomes. Lack of co-localization with EEA1 (early endosomal antigen in 1) in rat hippocampal neurons, fibroblasts, and human hNT neurons (Fig. 2D) excluded it from sorting endosomes. Co-localization of CLN3 with TGN38 further defined its presence in recycling endosomes. This was observed in primary rat (Fig. $2 E$ ) and human neurons (Fig. $2 F$ ), as well as human fibroblasts (Fig. 2G). Hence, CLN3 protein resides in the vicinity of the Golgi stacks (GRASP65), co-localizes with $\beta-1,4-$ GalT, an integral Golgi enzyme, and with TGN38, a marker for the trans Golgi network and recycling endosomes. Kremmidiotis et al. (22) previously identified CLN3 protein in cellular fractions corresponding to Golgi and PM.
Surface labeling experiments of nonpermeabilized rat neurons and human fibroblasts (Fig. $3 \mathrm{~A}$ ) with anti-CLN3 antibody at $4{ }^{\circ} \mathrm{C}$ corroborated that CLN3 is present at the PM. Co-localization of CLN3 with Gap43 (neuromodulin) in nonpermeabilized rat (Fig. $3 B$ ) and human neurons (Fig. 3C), and human fibroblasts (Fig. $3 D$ ), further confirmed its presence in the PM. This was corroborated in fibroblasts after co-transfection with pECFP-MEM (Gap43) vector and pEYFP-CLN3 vector (data not shown). CLN3 co-localized to the PM with TNF-R1, a death receptor, in nonpermeabilized human neurons (Fig. 3G). CLN3 also co-localized to the Golgi, together with TNF-R1, in permeabilized cells (results not shown). The TNF receptor, TNF-R1, is known to recycle between lipid rafts in the PM and Golgi. These findings imply that CLN3 protein recycles between Golgi and plasma membrane.

CLN3 uptake and trafficking from PM to Golgi via early recycling endosomes. To substantiate the presence of CLN3 in recycling endosomes, as suggested by co-localization with 

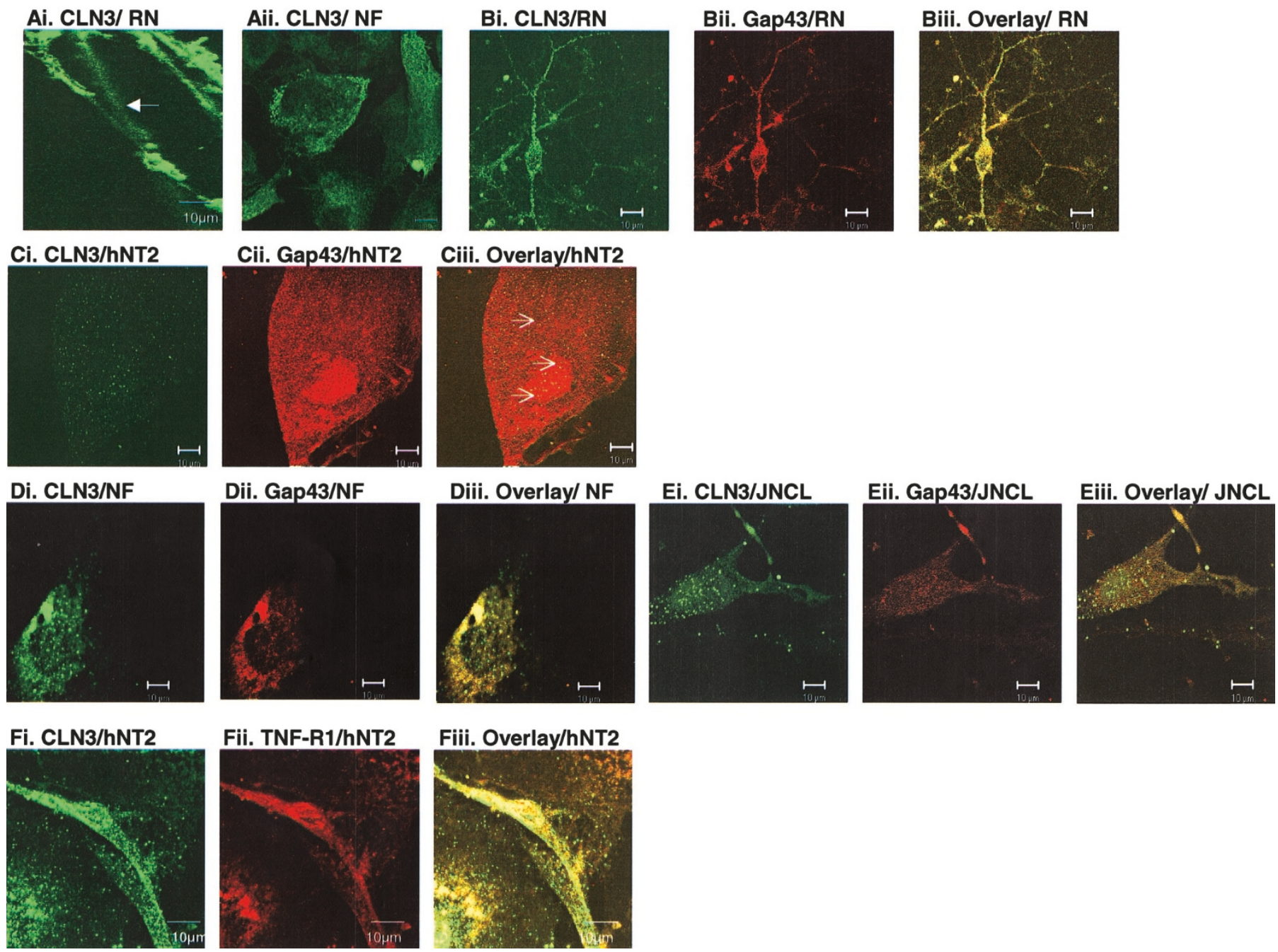

Figure 3. CLN3 and plasma membrane. (A) Surface labeling of CLN3 in primary rat hippocampus neurons $(i)$ and normal fibroblasts (ii). White arrow indicates presence of CLN3 in the plasma membrane. Co-localization of CLN3 and Gap43 proteins are shown in primary rat hippocampal neurons $(B)$, human hNT2 neurons $(C)$, normal fibroblasts $(D)$ and JNCL fibroblast $(E)$. (i) CLN3 is green. (ii) Gap43 is red. (iii) Overlay. Regions of co-localization are yellow. (F) Human hNT2 neurons dually labeled with CLN3 and TNF-R1 antibodies. (i) CLN3 is green. (ii) TNF-R1 is red. (iii) Overlay. Regions of co-localization are yellow. Cells were viewed at a magnification of $100 \times . N F$, normal fibroblasts; JNCL, JNCL fibroblasts; $h N T 2$, human neurons; $R N$, rat hippocampal neurons.

TGN38, co-localization with the Rab4, Rab5, and Rab11 proteins was examined. Rab11 is specific to early recycling endosomes, Rab4 to early endosomes involved in fast recycling of proteins and lipids, and Rab5 is a marker for early sorting endosomes $(35,36)$. CLN3 was minimally present in Rab5positive endosomes in rat hippocampal neurons (Fig. 4A). CLN3 partly co-localized with Rab4 to fast recycling endosomes in rat neurons and normal fibroblasts (Fig. 4, $B$ and $C$ ). Additionally, CLN3 partly co-localized with Rab11 in early recycling endosomes in normal fibroblasts (Fig. $4 E$ ) and rat neurons (Fig. 4G).

To observe movement of the CLN3 protein, live primary rat hippocampal neurons were transfected with the fluorescent YFP-CLN3 vector and then subjected to time-lapse microscopy. Rapid, dynamic movement of CLN3-positive endosomes was observed by time-lapse microscopy along axons in live primary rat neurons (video available at www.dbsr.duke/pub/ cln3). At any one moment, bidirectional movement of these endosomes was observed.
Internalization of CLN3 protein from the PM and velocity of endocytosis from PM to Golgi was approximated by determining the speed of internalization of CLN3, Rab4, and Rab11 proteins from the cell surface of human neurons, after incubating cells with the corresponding antibodies at $37^{\circ} \mathrm{C}$ for periods of $2,5,10$, and $15 \mathrm{~min}$ followed by cooling at $4^{\circ} \mathrm{C}$. As noted previously, surface labeling of the PM with CLN3 antibody is observed at $4{ }^{\circ} \mathrm{C}$. Maintaining cells at $37^{\circ} \mathrm{C}$ for different time periods promotes endocytosis, which is arrested by transfer of cells to $4^{\circ} \mathrm{C}$ followed by immediate fixation. CLN3 protein was internalized from the PM via Rab4- and Rab11-positive endosomes (Fig. 5). CLN3 internalization occurred before $2 \mathrm{~min}$ at $37^{\circ} \mathrm{C}$ and coincided with Rab4 internalization (Fig. $5 A$ ). Internalization proceeded further if cells were kept at $37^{\circ} \mathrm{C}$ for 5 min (Fig. 5B). CLN3p appeared in close proximity to the nucleus with less seen at the cell surface if the cells were kept at $37^{\circ} \mathrm{C}$ for $10 \mathrm{~min}$, before cooling and fixation (Fig. $5 \mathrm{C}$ ). The CLN3 protein was completely internalized by $15 \mathrm{~min}$ (Fig. $5 D$ ). Internalization of CLN3 protein was evident after 2 min at 


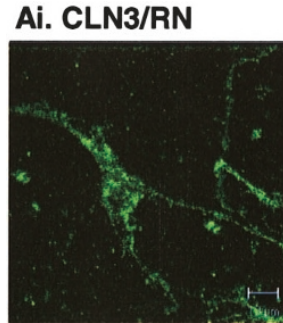

\section{Ci. CLN3/NF}

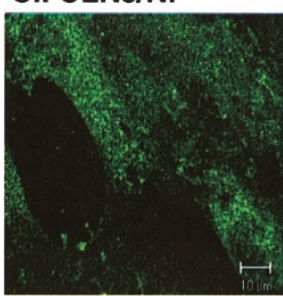

Ei. CLN3/NF

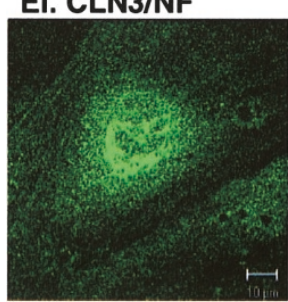

Gi. CLN3/RN

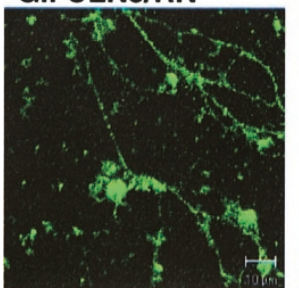

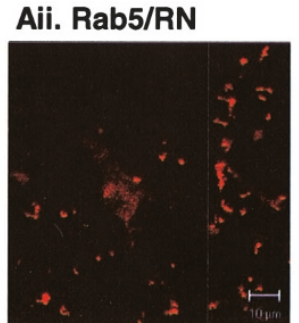

\section{Cii. Rab4/NF}

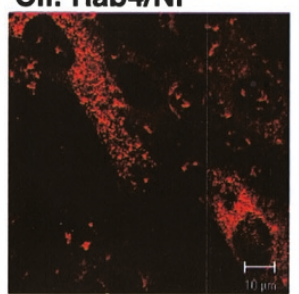

Eii. Rab11/NF

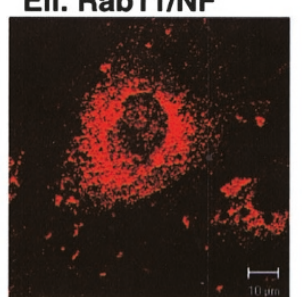

Gii. Rab11/RN

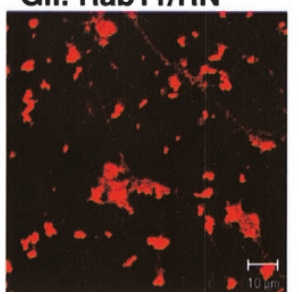

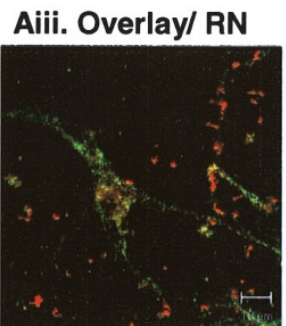

Cii. Overlay/ NF

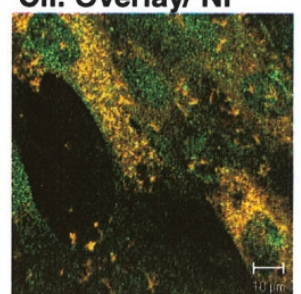

Eiii. Overlay/ NF

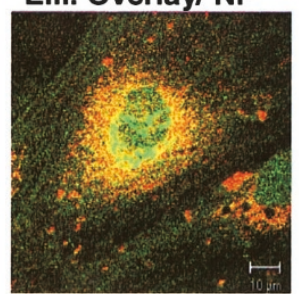

Giii. Overlay/ RN

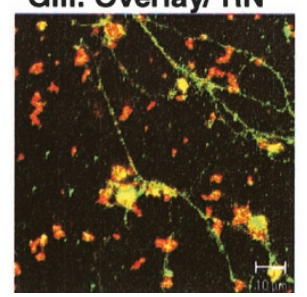

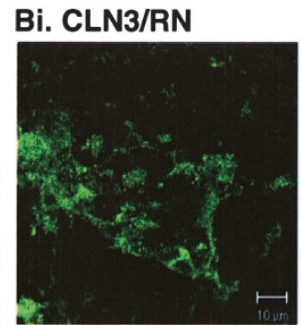

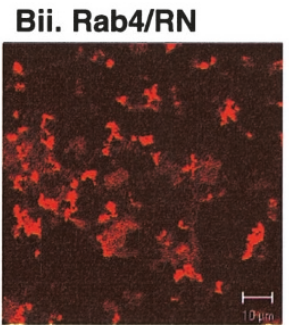

Di. CLN3/JNCL

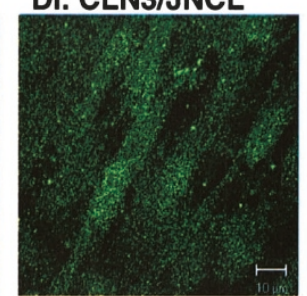

Dii. Rab4/JNCL

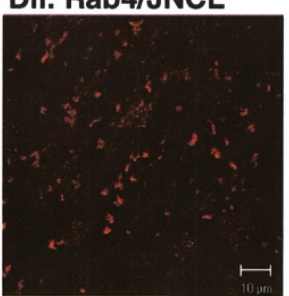

Fi. CLN3/JNCL
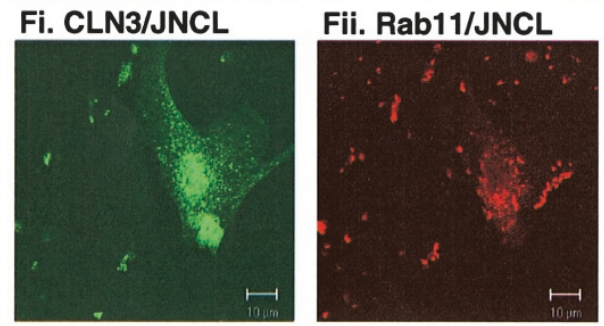

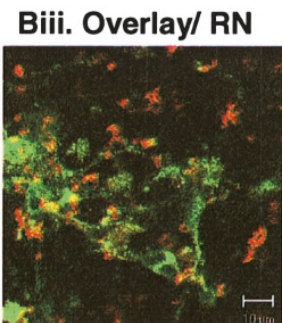

Diii. Overlay/ JNCL

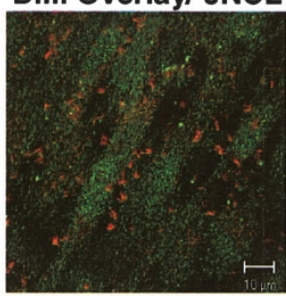

Fiii. Overlay/ JNCL

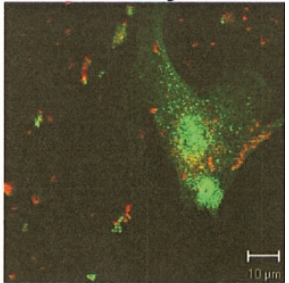

Figure 4. CLN3, Rab4, Rab11, and Rab5 proteins in endosomes. (A) Distribution of CLN3 and Rab5 proteins in rat neurons. (i) CLN3 is green. (ii) Rab5 is red. (iii) Overlay. Visualization of CLN3 and Rab4 proteins in rat neurons $(B)$, normal human fibroblasts $(C)$, and JNCL fibroblasts $(D)$. (i) CLN3 is green. (ii) Rab4 is red. (iii) Overlay. Regions of co-localization are yellow. Visualization of CLN3 and Rab11 proteins in normal human fibroblasts (E), JNCL fibroblasts $(F)$, and primary rat neurons $(G)$. (i) CLN3 is green. (ii) Rab11 is red. (iii) Overlay. Regions of co-localization are yellow. Cells were viewed at a magnification of $100 \times . N F$, normal fibroblasts; JNCL, JNCL fibroblasts; $h N T 2$, human neurons; $R N$, rat hippocampal neurons.

$37^{\circ} \mathrm{C}$, but internalization of Rab11 protein was not. Colocalization of Rab11 protein with CLN3 protein was first observed after $5 \mathrm{~min}$ of incubation at $37^{\circ} \mathrm{C}$ with antibodies (Fig. 5E). This reached a maximum after $10 \mathrm{~min}$ at $37^{\circ} \mathrm{C}$ (Fig. $5 F)$. CLN3p was completely internalized after $15 \mathrm{~min}$ of incubation with antibodies at $37^{\circ} \mathrm{C}$ (Fig. $5 G$ ). CLN3 protein co-localization with Rab11 protein was more extensive at the later time points. The temporal co-occurrence of CLN3 protein internalization and redistribution from cell surface to Golgi, together with that of Rab4 and Rab11 proteins, supports that CLN3 traffics from PM to Golgi via Rab4- and Rab11-positive endosomes.

CLN3 protein in lipid rafts and caveolae. To investigate whether the CLN3 motif, 291VYFAE295, is part of a GalCer binding domain structure, 28 amino acid residues (274-302) of the CLN3 sequence were transposed onto residues $1-28$ of the first $\alpha$-helix of $\beta$-amyloid precursor protein that contain amino acids 18VFFAE22 in the Protein Data Bank model, 1BJB, for comparative analysis of their structures. Topology was assessed by the Swiss-PROT PDB viewer and the MAGE/
PROBE molecule imaging program (available at: http:// kinemage.biochem.duke.edu/) (34). The resulting profile confirmed retained integrity of the backbone and no alteration of electrostatic interactions between neighboring residues. The CLN3 sequence also contained the requisite anchoring aromatic residues tyrosine (Y284) and phenylalanine (F287) essential for binding to GalCer (Fig. 6A). These analyses suggested the presence of a GalCer lipid raft binding domain in CLN3. Duallabeling studies with antibodies to CLN3 protein and the GPIanchored protein alkaline phosphatase, a known resident of lipid rafts, showed that CLN3 co-localized with alkaline phosphatase in human neurons (Fig. 6B), as well as normal fibroblasts (Fig. 6C). CLN3 also co-localized with caveolin-1, a protein specific for caveolae, in human fibroblasts (Fig. 6D). Immunoco-localization of CLN3 protein with lipid raft/caveolae marker proteins, and conformation of amino acids 274-302 to the structural GalCer lipid binding domain, strongly suggested the presence of CLN3 in lipid rafts. The subcellular localization of the two mutant CLN3 proteins CLN3 $\Delta 8-15$ and CLN3 $\Delta$ VYFAE is shown in Figure 7 and is discussed below. 


\section{Speed of Internalization of CLN3, Rab4 and Rab11 proteins in hNT2 neurons}
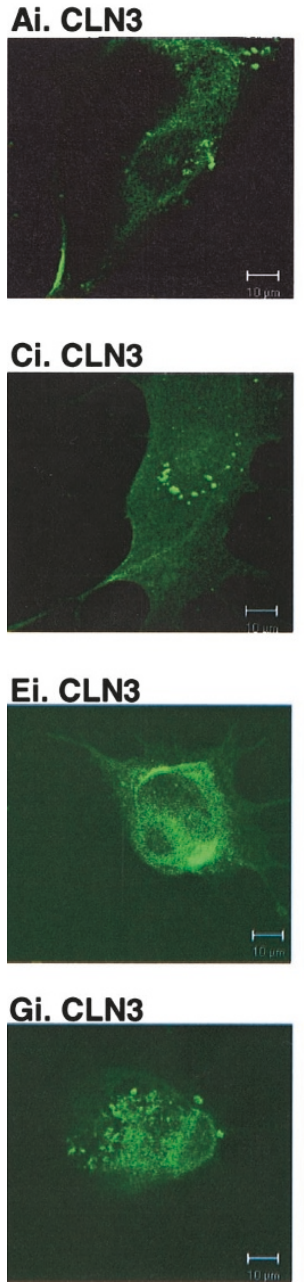
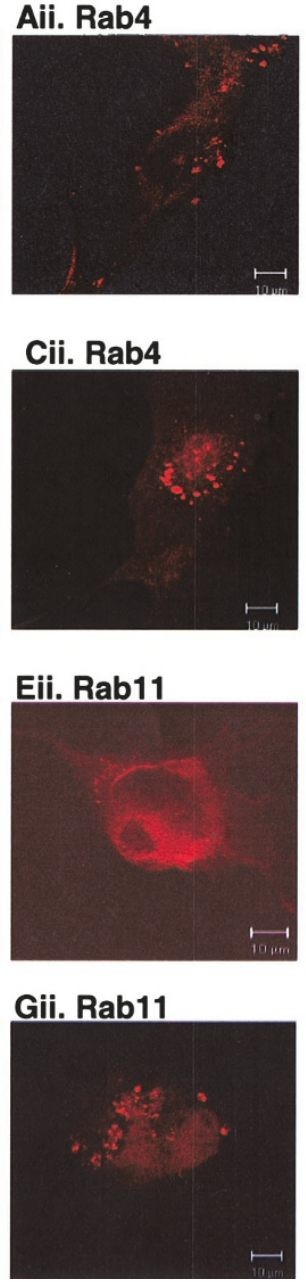
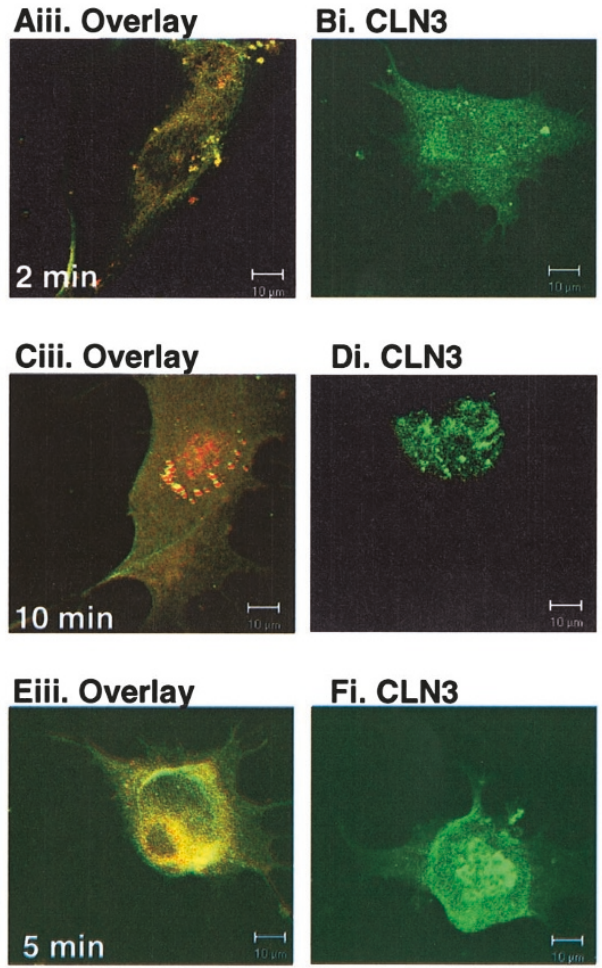
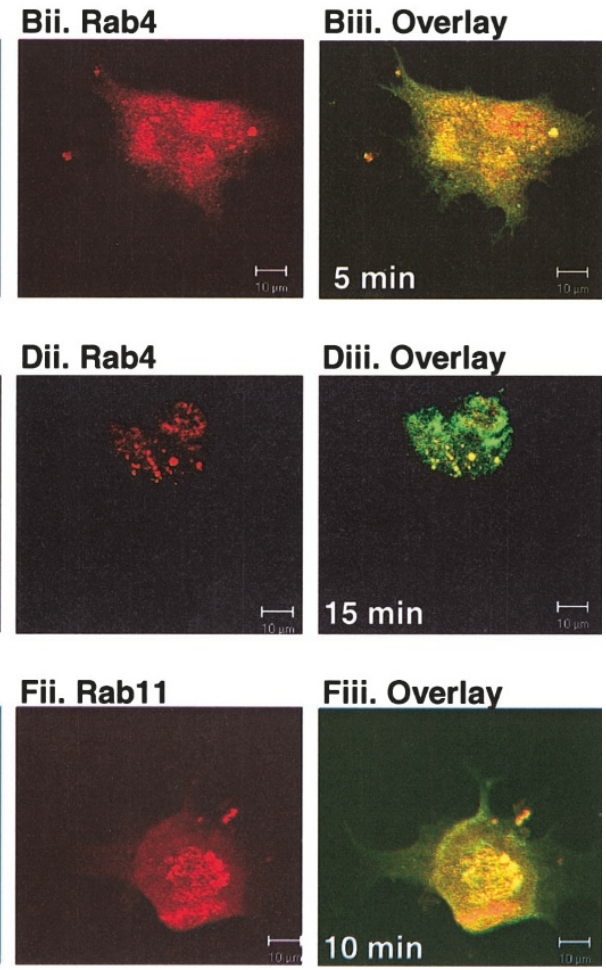

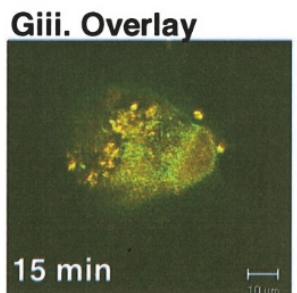

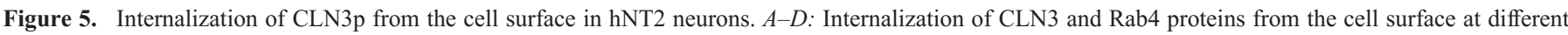

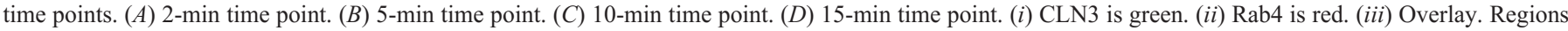

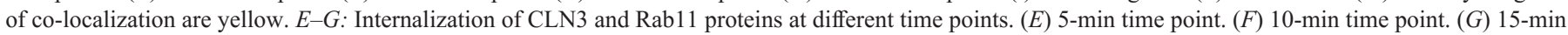
time point. (i) CLN3 is green. (ii) Rab11 is red. (iii) Overlay. Regions of co-localization appear yellow.

CLN3 and GalCer co-localized to distinct regions in the plasma membrane in nonpermeabilized normal cells (Fig. 8A). CLN3p co-localized with GalCer to Golgi in permeabilized normal cells (Fig. 8B).

Cellular mis-localization of mutant CLN3p. Determination of the subcellular localization of mutant CLN3 in patientderived cells was undertaken to understand the pathobiology leading up to lysosomal storage and accelerated apoptosis in JNCL. In all instances, JNCL or CLN3-deficient cells refer to fibroblasts derived from patients homozygous for the $1.02-\mathrm{kb}$ deletion in the genomic DNA.

Unlike wild-type protein, mutant CLN3 partially colocalized with cathepsin D (Fig. 1B), and with LAMP1 (Fig. $1 D$ ), in JNCL patient fibroblasts. This was confirmed by the existence of mutant CLN3 protein in Rab7-positive endosomes in JNCL fibroblasts (Fig. 1H). A substantial amount of mutant CLN3 protein co-localized with markers for the late endoso$\mathrm{mal} /$ lysosomal compartment in JNCL fibroblasts.

Mutant CLN3 also partly co-localized to Golgi, together with GRASP65 and TGN38 in JNCL patient fibroblasts, yet there were distinct differences observed in the structure of the Golgi in comparison with normal fibroblasts or neurons. Although mutant CLN3 was retained within the Golgi, the usually tubular Golgi structure was disrupted and fragmented in the majority of cells (Fig. $2 B$ ). We found that $53 \pm 11 \%$ of all JNCL fibroblasts showed fragmentation of the Golgi, compared with $0 \%$ in normal fibroblasts after $24 \mathrm{~h}(p<0.05)$. Additionally, although co-localization of mutant CLN3 protein with TGN38 was obvious, this was limited to the eccentric and perinuclear Golgi region (Fig. $2 H$ ). The diffuse pattern in normal fibroblasts and neurons seen with dual antibody labeling of TGN38 and CLN3, which are characteristic of recycling endosomes (Fig. 2, $F$ and $G$ ) was not observed in JNCL fibroblasts. The absence of mutant CLN3 from early recycling endosomes in patient-derived JNCL cells was confirmed by the failure of mutant CLN3 protein to co-localize with Rab4- (Fig. 4D) or Rab11-positive endosomes (Fig. 4F).

The absence of mutant CLN3 protein from recycling endosomes predicted its possible absence from the PM. Mutant CLN3 did not co-localize with Gap43 (Fig. 3F) to the cell 


\section{A. CLN3 GalCerLipid Raft binding Model}

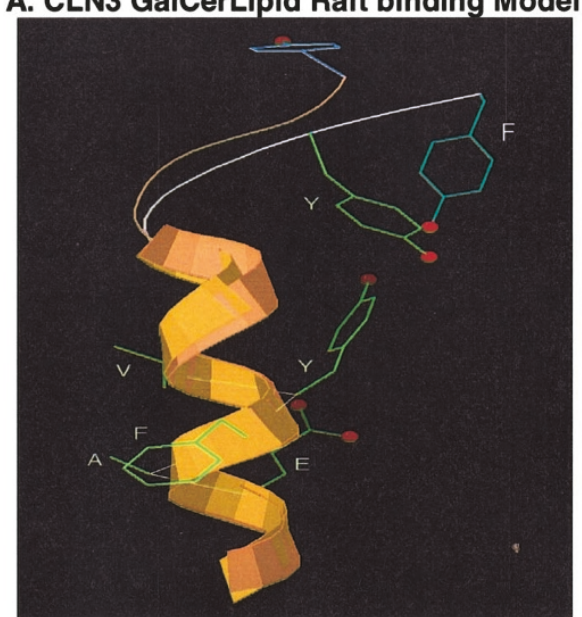

\section{Bi. CLN3/hNT2}

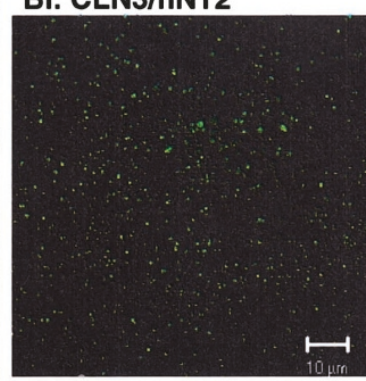

Bii. Alk. Phos/hNT2

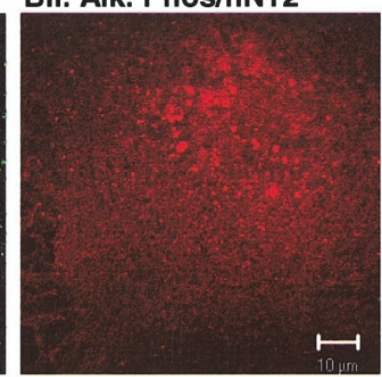

Biii. Overlay/ hNT2

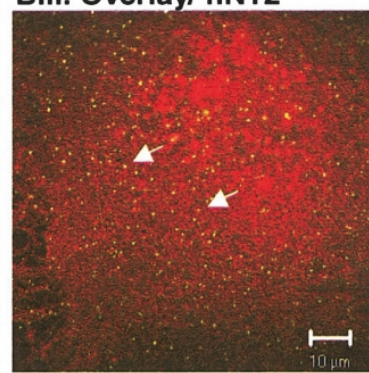

Ci. CLN3/NF

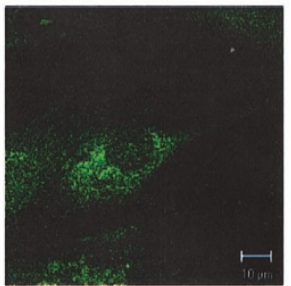

Di. CLN3/NF

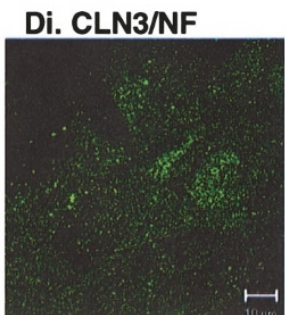

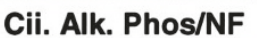

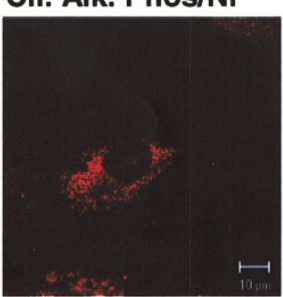

Dii. Caveolin 1/NF

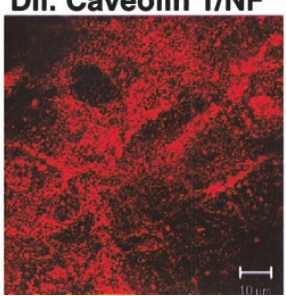

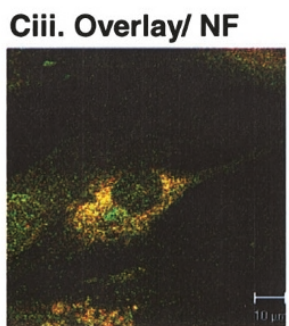

Diii. Overlay/ NF

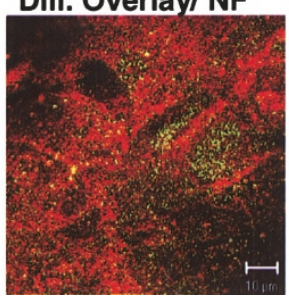

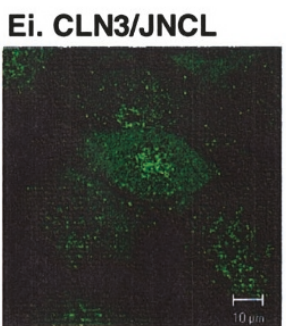

Fi. CLN3/JNCL

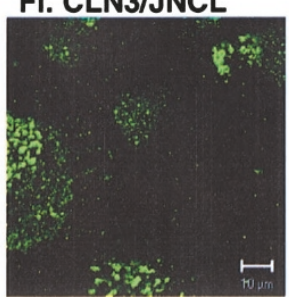

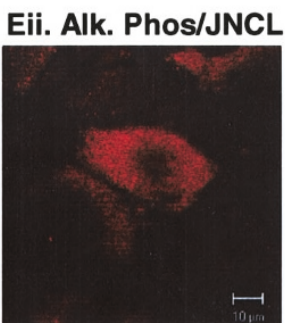

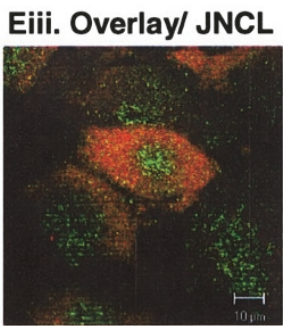

Fii. Caveolin 1/ JNCL Fiii. Overlay/ JNCL

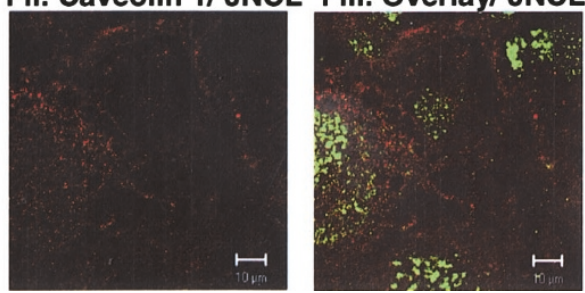

Figure 6. CLN3 and lipid rafts. (A) Hypothetical model of CLN3 residues 274-302. The CLN3 sequence is superimposed onto the $\beta$ amyloid model (PDB: 1BJB). The residues VYFAE are indicated. The white threading corresponds to the CLN3 model, and the peach threading outlines the $\beta$-amyloid model. In the CLN3 model tyrosine (Y284) and phenylalanine (F287) anchoring residues upstream of the VYFAE motif are indicated in green and teal, respectively. Double immuno-labeling with CLN3p and alkaline phosphatase antibodies in human hNT2 neurons $(B)$, normal human fibroblasts $(C)$, and JNCL fibroblasts $(D)$. $(i)$ CLN3 is green. (ii) Alkaline phosphatase is red. (iii) Overlay. Regions of co-localization are yellow, indicated by arrows. Double immuno-labeling of CLN3 and caveolin 1 proteins in normal human fibroblasts $(E)$ and JNCL or CLN3-deficient fibroblasts (F). (i) CLN3 is green. (ii) Caveolin 1 is red. (iii) Overlay. Cells were viewed at a magnification of $100 \times . N F$, normal fibroblasts; JNCL, JNCL fibroblasts; $h N T 2$, human neurons.

surface in JNCL patient fibroblasts. It was no surprise that mutant CLN3 protein, which lacks the amino acids responsible for the GalCer lipid raft binding structure, did not co-localize with the raft markers alkaline phosphatase (Fig. $6 E$ ) and caveolin-1 (Fig. 6F) and, hence, failed to appear in lipid rafts in JNCL cells.

Mutant protein CLN3 $\Delta 8-15 p$ co-localized with GRASP65 to fragmented Golgi regions (Fig. $7 A$ ). Similar to the endogenous mutant protein, $\mathrm{CLN} 3 \Delta 8-15 \mathrm{p}$ also mis-localized to late endosomes/lysosomes. This was confirmed by co-localization with Rab7 (Fig. 7B). CLN3 $\Delta 8-15 p$ did not co-localize with alkaline phosphatase to lipid rafts in the plasma membrane (Fig. 7C). The mutant protein CLN3 $\triangle$ VYFAEp co-localized with GRASP65 within fragmented Golgi regions (Fig. 7D). Minor areas of co-localization were observed between CLN3 3 VYFAE and Rab11 proteins (Fig. 7E), and CLN3 $\triangle$ VYFAE and alkaline phosphatase proteins (Fig. $7 F$ ).

Mutant CLN3p and GalCer. Endogenous mutant CLN3p and BODIPY-GalCer were absent from the plasma membrane in nonpermeabilized JNCL fibroblasts (Fig. 8C). Mutant CLN3p and GalCer showed co-localization restricted to the Golgi in permeabilized JNCL cells (Fig. 8D). The introduction of wild-type CLN3 cDNA into JNCL cells corrected the defect in GalCer and CLN3 localization: both CLN3p and GalCer were now present at the PM (Fig. $8 E$ ) and in the Golgi (Fig. $8 F$ ), similar to wild-type cells.

The mutant CLN3 construct CLN3 $\Delta 8-15 p$ co-localized with GalCer in a manner similar to that found with the endogenous mutant CLN3 protein, CLN3(1-153*28). Neither CLN3 $\Delta 8-15 p$ nor GalCer were present at the PM in nonpermeabilized JNCL cells (Fig. 8G), but were retained within the Golgi (Fig. 8H). CLN3 $\triangle$ VYFAEp and GalCer demonstrated minor co-localization at the plasma membrane in nonpermeabilized JNCL cells. CLN3 $\triangle$ VYFAEp and GalCer were present primarily in the Golgi in permeabilized cells (data not shown).

CLN3 and sphingolipids. The levels of a number of sphingolipids were measured in CLN3-deficient and normal cells following metabolic labeling with ${ }^{14} \mathrm{C}$-palmitate. The levels of 


\section{Sub-cellular localization of CLN3 $\Delta 8-15$ and CLN3}
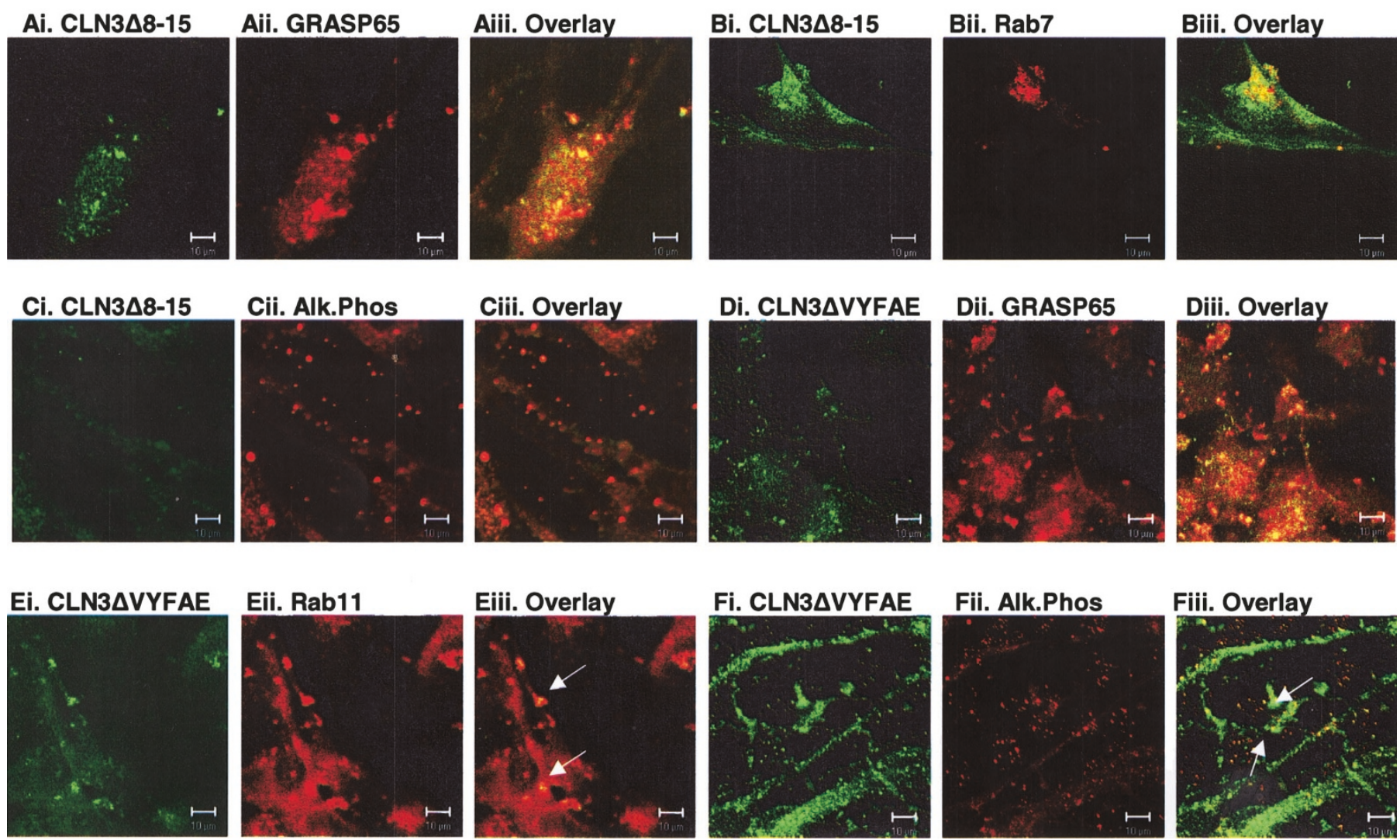

Figure 7. Localization of CLN3 mutant proteins CLN3 $38-15$ and CLN3 3 VYFAE in JNCL fibroblasts. ( $A$ ) Co-localization of CLN3 $88-15$ and GRASP65. $(i)$ CLN3 $\Delta 8-15$ is green. (ii) GRASP65 is red. (iii) Overlay. Regions of co-localization are yellow. (B) Co-localization of CLN3 $\Delta 8-15$ and Rab7. (i) CLN3 $\Delta 8-15$ is green. (ii) Rab7 is red. (iii) Overlay. Regions of co-localization are yellow. (C) Co-localization of CLN3 $\Delta 8-15$ and alkaline phosphatase. (i) CLN3 $\Delta 8-15$ is green. (ii) Alkaline phosphatase is red. (iii) Overlay. (D) Co-localization of CLN3 $\triangle$ VYFAE and GRASP65. (i) CLN3 co-localization are yellow. (E) Co-localization of CLN3 $\triangle$ VYFAE and Rab7. (i) CLN3 VVYFAE is green. (ii) Rab7 is red. (iii) Overlay. Regions of co-localization are yellow (indicated by white arrow). (F) Co-localization of CLN3 $\mathrm{VYYFAE}$ and alkaline phosphatase. (i) CLN3 $\Delta$ VYFAE is green. (ii) Alkaline phosphatase is red. (iii) Overlay. Regions of co-localization are yellow (indicated by white arrow).

ceramide (79\%), sphingomyelin (26\%), GalCer $(27 \%)$, GluCer $(32 \%)$, globoside $(32 \%)$, and CTH $(4 \%)$ were up-regulated in JNCL compared with normal cells (Fig. 9a). As far as ceramide is concerned, introducing CLN3 into JNCL cells lowered levels, and more importantly, over-expression of CLN3 in normal cells reduced ceramide even further (Fig. 9b).

\section{DISCUSSION}

In this study, we establish 1) that wild-type CLN3 is present within the Golgi complex and traffics to lipid rafts in the PM via Rab4- and Rab11-positive endosomes, and 2) that CLN3 may be required for the efficient transport of GalCer to lipid rafts. Mutant CLN3 protein is retained within a disrupted Golgi, and not within the ER as suggested by others $(18,19$, 37). Golgi fragmentation and subsequent perinuclear distribution in neurons are histologic hallmarks of many neurodegenerative diseases including Alzheimer's, Creutzfeldt-Jakob, and prionic protein diseases (38). CLN3-deficient cells exhibit these findings. Caspase-mediated cleavage of GRASP65 is an initial event in Golgi fragmentation, typically seen in the late stages of apoptosis (39). Fragmentation of the Golgi in JNCL cells is consistent with previous reports linking defects in the CLN3 gene to apoptosis $(11,13)$ and was observed in JNCL fibroblasts (Fig. 2B).

The CLN3 protein sequence contains a number of tri- or dileucine motifs (LL or LI) that play important roles in the targeting of proteins to the PM, clathrin-coated pits, and anchoring to microvilli $(18,28,40-42)$. Presence of CLN3 at the PM was confirmed by surface labeling, co-localization of CLN3 protein with neuromodulin or Gap43, and TNF-R1 in nonpermeabilized cells.

The endosomal system retrieves proteins from the PM and the trans Golgi network (TGN) before delivery to a large pool of early endosomes. CLN3 protein co-localized with TGN38 to the Golgi, and CLN3, like TGN38, recycles between the PM and Golgi via early recycling endosomes (43). Co-localization of CLN3p with Rab4- and Rab-11-positive endosomes supports this (44). The absence of co-localization of wild-type 


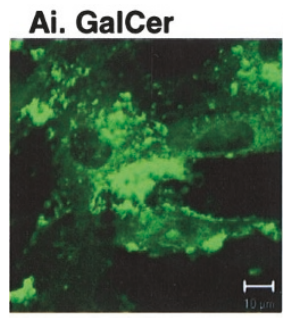

Ci. GalCer

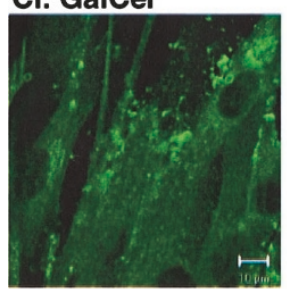

Ei. GalCer

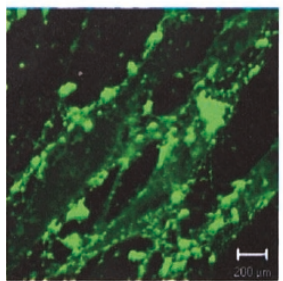

Gi. GalCer

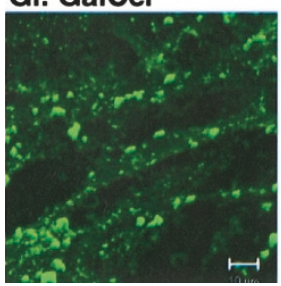

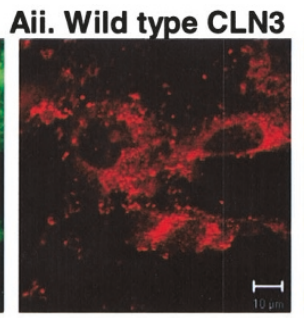

Cii Mutant CLN3p

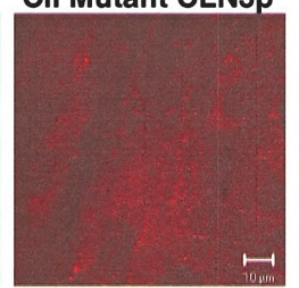

Ei. +CLN3(1-15)

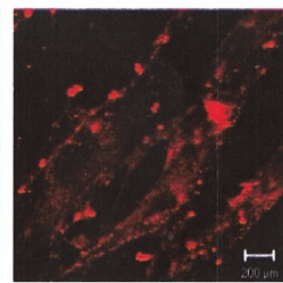

Gii. +CLN3 $\Delta 8-15$

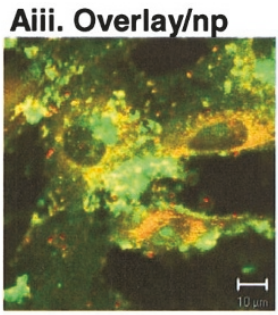

Ciii. Overlay/np

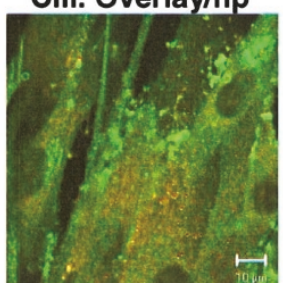

Eiii. Overlay/np

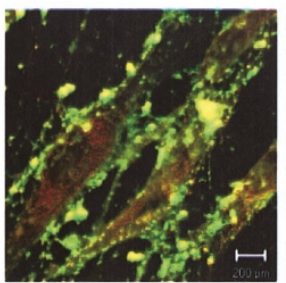

Giii. Overlay/np
Bi. GalCer

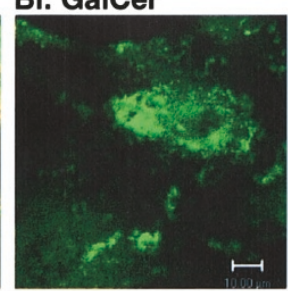

Di. GalCer

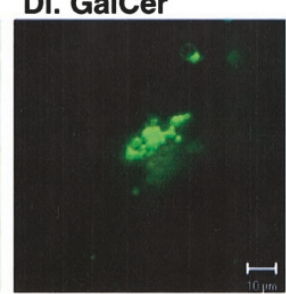

Fi. GalCer

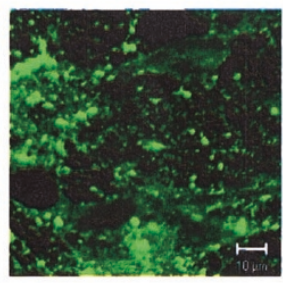

Hi. GalCer

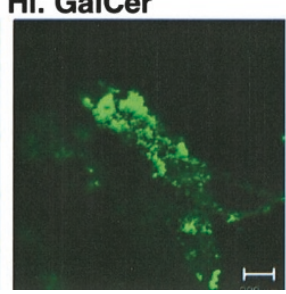

Bii. Wild type CLN3 Biii.Overlay/p

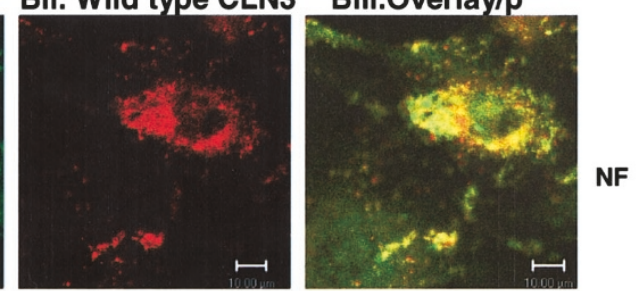

Dii. Mutant CLN3p

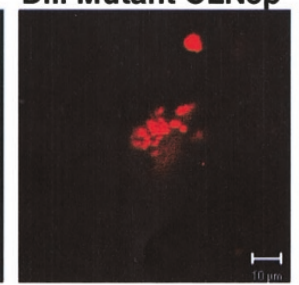

Fii. +CLN3(1-15)

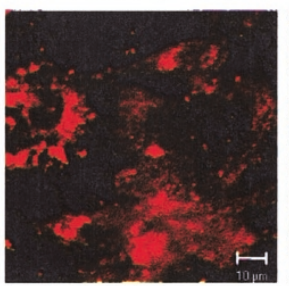

Hii. +CLN3 $\Delta$ 8-15
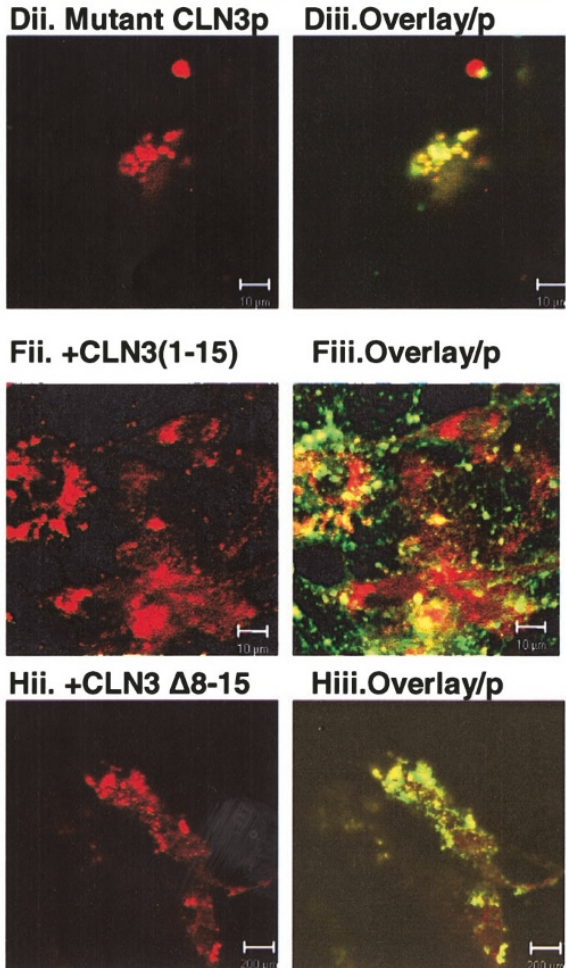

JNCL
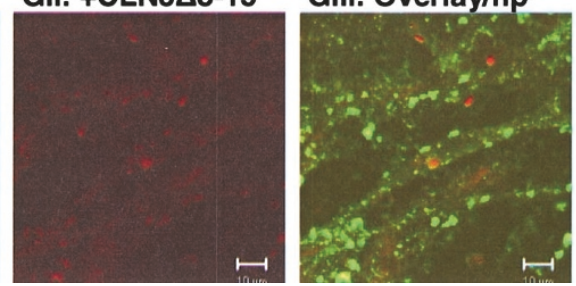

Figure 8. CLN3p and galactosylceramide. Co-localization of endogenous CLN3p and the lipid GalCer in nonpermeabilized $(A)$ and permeabilized $(B)$ normal fibroblasts. ( ) GalCer is green. (ii) CLN3p is red. (iii) Overlay. Regions of co-localization are shown in yellow. Dual labeling of endogenous mutant CLN3p and GalCer in nonpermeabilized $(C)$ and permeabilized $(D)$ JNCL fibroblasts. Co-localization of the introduced intact CLN3 (1-15) protein and GalCer in nonpermeabilized $(E)$ and permeabilized $(F)$ JNCL fibroblasts. (i) GalCer is green. (ii) CLN3p is red. (iii) Overlay. Regions of co-localization are shown in yellow. Co-localization of expressed mutant CLN3 protein CLN3 $\Delta 8-15$ and GalCer in nonpermeabilized $(G)$ and permeabilized $(H)$ JNCL fibroblasts. $N F$, normal fibroblasts; $J N C L$, JNCL fibroblasts; $n p$, nonpermeabilized cells; $p$, permeabilized cells.

CLN3 protein with EEA1 was surprising. EEA1 is an effector for Rab5, and Rab5 is involved in early sorting of proteins from the plasma membrane to early recycling endosomes, and also to late endosomes/lysosomes via a separate route $(45,46)$. Co-localization of CLN3 with Rab11 places CLN3 in recycling endosomes and the TGN (47-50).

CLN3 protein internalization studies confirmed that CLN3 entered the cell from the cell surface within Rab4-positive endosomes at an early time point. CLN3 presence within Rab11-positive endosomes occurred later than within Rab4positive endosomes. Co-localization of CLN3 with Rab4 decreased, whereas co-localization of CLN3 with Rab11 increased over a $15-\mathrm{min}$ period. This supports the notion that the CLN3 protein may undergo or be involved in fast and early recycling from the PM to the TGN, and that CLN3 trafficking back to the Golgi is complete within $15 \mathrm{~min}$ of endocytosis from the cell surface. The lack of CLN3-positive fluorescent signals from the cell periphery and extensions at $15 \mathrm{~min}$ supports this (Fig. 5, $D$ and $G$ ). The intact CLN3 protein therefore traffics between PM and the Golgi via Rab4- and Rab11-positive endosomes.

CLN3p is highly mobile and traffics along rat neuronal processes in both directions as shown by time-lapse microscopy (video 1). The simultaneous observation of CLN3 protein moving in both directions supports the concept of recycling. CLN3 protein trafficking appeared less dynamic in fibroblasts than it did in neuronal cells (data not shown). The rapid movement of CLN3 observed in neurons may have particular significance, and may explain why the brunt of the pathology is limited to neurons and photoreceptors in JNCL.

Mutant CLN3p did not co-localize to Rab4- and Rab11positive recycling endosomes, but did show some colocalization with Rab7, a marker for the late endosome/ lysosomal system. One must conclude that endocytic recycling of mutant CLN3p was abnormal. Defective endocytic recycling has been implicated in a number of neurodegenerative diseases, such as Niemann-Pick, type C (51). This may be a common theme in JNCL and perhaps other NCL disorders with 

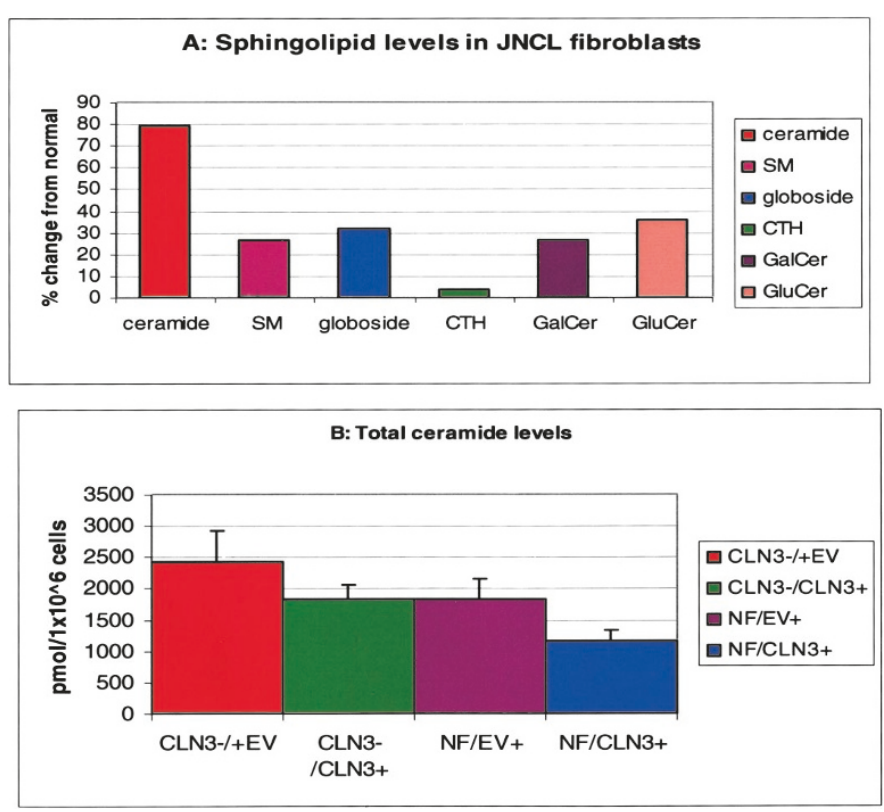

Figure 9. Sphingolipids in JNCL and normal fibroblasts. $(A){ }^{14} \mathrm{C}$-palmitate labeling. Ceramide or Cer ( $\uparrow 79 \%)$, sphingomyelin or SM levels ( $\uparrow 26 \%)$, galactosylceramide or GalCer $(\uparrow 27 \%)$, glucosylceramide or GluCer ( $\uparrow 32 \%)$, GB4 or globoside ( $\uparrow 32 \%)$, and ceramide trihexoside or $\mathrm{CTH}(\uparrow 4 \%)$ levels in JNCL fibroblasts. Measurements were normalized to proteins and are shown as percentage change from normal controls. $(B)$ Ceramide levels. Ceramide levels in JNCL cells transfected with an empty vector control or CLN3-/EV+ (red bar). Ceramide levels in JNCL cells transfected with intact CLN3 indicated as CLN3-/CLN3 + or CLN3 (1-15) (green bar). Ceramide levels in normal fibroblasts transfected with an empty vector or NF/EV+ (purple bar). Ceramide levels in normal fibroblasts transfected with intact CLN3 indicated as NF/CLN3+ or CLN3 (1-15) (blue bar).

membrane protein defects and associated dysregulation of lipid/sphingolipid metabolism.

The CLN3 protein structurally fits the GalCer/lipid raft binding domain model, as discussed above (Fig. 6A). Colocalization of CLN3 with alkaline phosphatase and caveolin-1 in human neurons and fibroblasts, respectively, strongly suggests that lipid rafts and caveolae are enriched with CLN3. Entry of certain proteins into cells via lipid rafts and caveolae may contribute to the pathology of Alzheimer's disease and prionic diseases. Lipid rafts may also act as portals of entry for pathogens such as the HIV virus, and cholera and Anthrax toxins $(27,52,53)$. The HIV virus interacts with GalCer in lipid rafts of neurons and epithelial cells via the V3 loop of its surface glycoprotein, gp120 $(54,55)$. The interaction of the $\beta$-amyloid, $\operatorname{PrP}^{\mathrm{sc}}$, and gp120 proteins with lipid rafts is mediated via a GalCer binding domain (23). Synthetic peptides derived from the V3-like domain of infectious $\mathrm{PrP}^{\mathrm{sc}}$ interact with GalCer and SM, two major constituents of lipid rafts (27, 56). Naturally occurring mutations within the structurally conserved helix-turn-helix GalCer binding domain in the infectious $\operatorname{PrP}^{\mathrm{sc}}(\mathrm{E} 220 \mathrm{~K})$ and $\beta$-amyloid (E22Q, E22K) proteins result in increased apoptosis and clinical disease $(26,27)$. The analogous, naturally occurring CLN3 mutation E295K also results in increased apoptosis and disease (25). Additionally, CLN3, normal prionic protein $\operatorname{PrP}^{\mathrm{c}}, \beta$-amyloid, and gp120 proteins all harbor the important tyrosine and phenylalanine residues necessary for binding to sugar rings of complex carbohydrates. It has been suggested that these disorders could be treated by altering the composition and lipid stoichiometry of lipid rafts by depletion of cholesterol $(52,57)$. Manipulation of lipid raft composition may be an avenue for development of novel therapies for JNCL and other diseases where lipid rafts are implicated.

In more than $80 \%$ of JNCL patients, the CLN3 protein lacks amino acids 153-438 and, therefore, the GalCer lipid raft binding domain that harbors the 291VYFAE295 motif and the additional tyrosine and phenylalanine residues necessary for binding to sugar residues. Disruption of this domain probably prevents mutant CLN3 protein from trafficking normally and binding correctly to lipid rafts. Co-localization of CLN3p and GalCer is demonstrated in the Golgi and at the plasma membrane in normal cells. Neither mutant CLN3p nor GalCer are present at the PM in JNCL cells. They are retained within a fragmented Golgi. The introduction of intact CLN3 into JNCL cells restored the presence of CLN3p and GalCer to the PM, supporting the requirement of the GalCer binding domain for efficient transport and trafficking of these molecules from Golgi to PM.

The mutant CLN3 $\Delta 8-15 \mathrm{p}$, which lacks the GalCer lipid raft binding domain, co-localized in a manner similar to that of endogenous mutant protein. Absence of both GalCer and CLN3 $\Delta 8-15 p$ at the PM supports the notion that CLN3p with an intact GalCer binding domain is required for delivery of GalCer to lipid rafts.

The GalCer binding domain is made up of multiple elements, one of which is the VYFAE motif. The aromatic anchoring residues Y284 and F287 that are upstream of this motif play a role in promoting binding of CLN3 to GalCer carbohydrate moieties, and both are intact in the mutant CLN3 $\triangle$ VYFAE construct. Therefore, deletion of the minimal motif, 291VYFAE295, within CLN3p may result in much looser, but not totally absent, binding of CLN3 $\triangle$ VYFAEp to GalCer. This accounts for the minimal co-localization of CLN3 $\triangle$ VYFAE with alkaline phosphatase and GalCer in lipid rafts, and of GalCer and CLN3 $\triangle$ VYFAE in Rab11-positive endosomes. JNCL patients with the E295K mutation manifest a protracted form of JNCL, where onset is delayed and symptoms are less severe (25).

The pro-apoptotic lipid second messenger, ceramide, is the building block for a number of sphingolipids found in lipid rafts, including SM, GalCer, CTH, globoside, and glucosylceramide (GluCer) (58). Ceramide is synthesized in the ER via de novo synthesis, and in the Golgi, the PM, and mitochondrial membrane via hydrolysis of sphingomyelin. GluCer is synthesized on the cytosolic face of the Golgi, SM is synthesized in the Golgi lumen, and GalCer is synthesized in the lumen of the ER in epithelial and neuronal cells (59). Sphingolipids are synthesized in the ER or Golgi, and are translocated to the PM via endosomes (60). There is evidence that lipid rafts destined for the PM membrane originate in the Golgi where many of their sphingolipid constituents are synthesized $(28,61)$. Sphingolipids constitute $20-50 \%$ of the polar lipids within the PM, with GalCer being a major sphingolipid constituent of myelin and lipid rafts in neuronal cells (62). Moreover, lipid raft 
function is also dependent on specific stoichiometry of its sphingolipid content. The close physical proximity of CLN3 protein and GalCer in Golgi and also in lipid rafts in the PM enhances the belief that their functions are interrelated. Failure of binding of mutant CLN3 protein to GalCer in nascent lipid rafts or in the Golgi may result in perturbation of GalCer and other sphingolipid levels and, therefore, impaired raft function and dysregulated apoptosis.

It had previously been determined that ceramide was increased by $80 \%$, with an associated $20 \%$ increase of sphingomyelin levels in CLN3-deficient fibroblasts (33). GalCer, GluCer, globoside, and CTH levels were also increased. Introducing CLN3 into JNCL fibroblasts reduced ceramide levels to normal, and CLN3p overexpression in normal cells reduced ceramide levels even further, suggesting that CLN3 may play a role in the regulation of sphingolipid levels.

The observed pathobiology in CLN3 deficiency could be explained in the following manner: Mutant CLN3 lacking the GalCer binding domain appears in late endosomes/lysosomes, and fails to traffic within early recycling endosomes between Golgi and PM. This suggests that endocytic recycling is defective in JNCL. Failure of mutant CLN3 to bind to GalCer for delivery to lipid rafts may lead to impaired lipid raft function and, hence, impaired anti-apoptotic signaling in JNCL. Inability of CLN3 protein to bind to GalCer at its site of synthesis in the Golgi may lead to perturbation in the levels of GalCer and other sphingolipids, including ceramide, globoside, GluCer, and $\mathrm{CTH}$.

The data generated establishes a plausible link between a structural domain within wild-type CLN3 protein and its subcellular localization in lipid rafts, Golgi, and recycling endosomes. This work also provides a credible theory linking the subcellular mis-localization of mutant CLN3 protein, observed perturbations in sphingolipid levels and increased apoptosis seen in CLN3-deficient states.

Acknowledgments. The authors thank Jane Richardson for her help with molecular protein modeling and contributions to the manuscript, Dr. Terry Oas for his recommendations concerning protein modeling, Dr. Francis Barr for supplying the GRASP65 antibody, and Dr. Mike Ehler for the EEA1 antibody. We also thank Dr. Yusuf Hannun for valuable suggestions and critical review of the manuscript.

\section{REFERENCES}

1. Boustany R-M 1996 Batten disease or neuronal ceroid lipofuscinosis. In: Vinken PJ, Bruyn GW (eds) Neurodystrophies and Neurolipidoses. Elsevier, New York, pp 671-700

2. Zhong N 2000 Neuronal ceroid lipofuscinoses and possible pathogenic mechanism. Mol Genet Metab 71:195-206

3. Schmechel DE 1999 Apoptosis in neurodegenerative disorders. In Hannun Y, Boustany R-M (eds) Apoptosis in Neurobiology. CRC Press, Boca Raton, pp 29-30

4. Kida E, Golabek AA, Wisniewski KE 2001 Cellular pathology and pathogenic aspects of neuronal ceroid lipofuscinoses. Adv Genet 45:35-68

5. Guo WX, Mao C, Obeid LM, Boustany RM 1999 A disrupted homolog of the CLN3 or juvenile neuronal ceroid lipofuscinosis gene in Sacchromyces cerevisiae. Cell Mol Neurobiol 19:671-680

6. Holopainen JM, Saarikoski J, Kinnunen PK, Jarvela I 2001 Elevated lysosomal pH in neuronal ceroid lipofuscinoses (NCLs). Eur J Biochem 268:5851-5856

7. Dhar S, Bitting RL, Rylova SN, Jansen PJ, Lockhart E, Koeberl DD, Amalfitano A, Boustany R-M 2002 Flupirtine blocks apoptosis in batten patient lymphoblasts and in human postmitotic CLN3- and CLN2-deficient neurons. Ann Neurol 51:448-466
8. Kieseier BC, Wisniewski KE, Park E, Schuller-Levis G, Mehta PD, Goebel HH 1997 Leukocytes in neuronal ceroid-lipofuscinoses: function and apoptosis. Brain Dev 19:317-322

9. Lane SC, Jolly RD, Schmechel DE, Alroy J, Boustany RM 1996 Apoptosis as the mechanism of neurodegeneration in Batten's disease. J Neurochem 67:677-683

10. Pane MA, Puranam KL, Boustany RM 1999 Expression of $\operatorname{cln} 3$ in human NT2 neuronal precursor cells and neonatal rat brain. Pediatr Res 46:367-374

11. Persaud-Sawin DA, VanDongen A, Boustany RM 2002 Motifs within the CLN3 protein: modulation of cell growth rates and apoptosis. Hum Mol Genet 11:21292142

12. Puranam K, Qian WH, Nikbakht K, Venable M, Obeid L, Hannun Y, Boustany RM 1997 Upregulation of Bcl-2 and elevation of ceramide in Batten disease. Neuropediatrics 28:37-41

13. Puranam KL, Guo WX, Qian WH, Nikbakht K, Boustany RM 1999 CLN3 defines a novel antiapoptotic pathway operative in neurodegeneration and mediated by ceramide. Mol Genet Metab 66:294-308

14. Cutler RG, Pedersen WA, Camandola S, Rothstein JD, Mattson MP 2002 Evidence that accumulation of ceramides and cholesterol eters mediates oxidative stressinduced death of motor neurons in amyotrophic lateral sclerosis. Ann Neurol 52:448 457

15. Obeid L, Hannun Y 2003 Ceramide, stress, and a "LAG" in aging. Sci Aging Knowledge Environ 2003:PE27

16. Katz ML, Gao CL, Prabhakaram M, Shibuya H, Liu PC, Johnson GS 1997 Immunochemical localization of the Batten disease (CLN3) protein in retina. Invest Ophthalmol Vis Sci 38:2375-2386

17. Pearce DA, Ferea T, Nosel SA, Das B, Sherman F 1999 Action of BTN1, the yeast ortholog of the gene mutated in Batten Disease. Nat Genet 22:55-58

18. Jarvela I, Sainio M, Rantmaki T, Olkkonen VM, Carpen O, Peltonen L, Janes RW 1998 Biosynthesis and intracellular targeting of the CLN3 protein defective in Batten disease. Hum Mol Genet 7:285-291

19. Haskell RE, Carr CJ, Pearce DA, Bennett MJ, Davidson BL 2000 Batten disease: evaluation of CLN3 mutations on protein localization and function. Hum Mol Genet 9:735-744

20. Margraf LR, Boriack RL, Routheut AA, Cuppen I, Alhilali L, Bennett CJ, Bennett MJ 1999 Tissue expression and subcellular localization of CLN3, the Batten disease protein. Mol Genet Metab 66:283-289

21. Luiro K, Kopra O, Lehtovirta M, Jalanko A 2001 CLN3 protein is targeted to neuronal synapses but excluded from synaptic vesicles: new clues to Batten disease. Hum Mol Genet 10:2123-2131

22. Kremmidiotis G, Lensink IL, Bilton RL, Woollatt E, Chataway TK, Sutherland GR, Callen DF 1999 The Batten disease gene product (CLN3p) is a Golgi integral membrane protein. Hum Mol Genet 8:523-531

23. Mahfoud R, Garmy N, Maresca M, Yahi N, Puigserver A, Fantini J 2002 Identification of a common sphingolipid-binding domain in Alzheimer, prion, and HIV-1 proteins. J Biol Chem 277:11292-11296

24. Sutton JM, Chow-Worn O, Spaven L, SilmanNj, Hallis B, Shone CC 2001 Tyrosine1290 of tetanus neurotoxin plays a key role in its binding to gangliosides and functional binding to neurones. FEBS Lett 493:45-49

25. Zhong N, Wisniewski KE, Kaczmarski AL, Ju W, Xu WM, Xu WW, Mclendon L, Liu B, Kaczmarski W, Sklower Brooks SS, Brown WT 1998 Molecular screening of Batten disease: identification of a missense mutation (E295K) in the CLN3 gene. Hum Genet 102:57-62

26. Miravalle L, Tokuda T, Chiarle R, Giaccone G, Bugiani O, Tagliavini F, Frangione B, Ghiso J 2000 Substitutions at codon 22 of Alzheimer's abeta peptide induce diverse conformational changes and apoptotic effects in human cerebral endothelial cells. J Biol Chem 275:27110-27116

27. Fantini J, Garmy N, Mahfoud R, Yahi N 2002 Lipid rafts: structure, function and role in HIV, Alzheimer's and prion diseases. Expert Rev Mol Med 2002:1-22

28. Simons K, Ikonen E 1997 Functional rafts in cell membranes. Nature 387:569-572

29. Zacharias DA, Violin JD, Newton AC, Tsien RY 2002 Partitioning of lipid-modified monomeric GFPs into membrane microdomains of live cells. Science 296:913-916

30. Janes RW, Munroe PB, Mitchison HM, Gardiner RM, Mole SE, Wallace BA 1996 A model for Batten disease protein CLN3: functional implications from homology and mutations. FEBS Lett 399:75-77

31. Pullarkat RK, Morris GN 1997 Farnesylation of Batten disease CLN3 protein. Neuropediatrics 28:42-44

32. Pullarkat RK, Kim KS, Sklower SL, Patel VK 1998 Oligosaccharyl diphosphodolichols in the ceroid-lipofuscinoses. Am J Med Gen Suppl 5:243-251

33. Rylova SN, Amalfitano A, Persaud-Sawin DA, Guo WX, Chang J, Jansen PJ, Proia $\mathrm{AD}$, Boustany RM 2002 The CLN3 gene is a novel molecular target for cancer drug discovery. Cancer Res 62:801-808

34. Word MJ, Bateman Jr RC, Presley BK, Lovell SC, Richardson DC 2000 Exploring steric constraints on protein mutations using MAGE/PROBE. Protein Sci 9:22512259

35. Cormont M, Mari M, Galmiche A, Hofman P, Le Marchand-Brustel Y 2001 A FYVE-finger-containing protein, Rabip4, is a Rab4 effector involved in early endosomal traffic. Proc Nat Acad Sci U S A 98:1637-1642

36. Sonnichsen B, De Renzis S, Nielsen E, Rietdorf J, Zerial M 2000 Distinct membrane domains on endosomes in the recycling pathway visualized by multicolor imaging of Rab4, Rab5 and Rab11. J Cell Biol 149:901-914

37. Jarvela I, Lehtovirta M, Tikkanen R, Kyttala A, Jalanko A 1999 Defective intracellular transport of CLN3 is the molecular basis of Batten disease (JNCL). Hum Mol Genet 8:1091-1098

38. Sakurai A, Okamoto K, Fujita Y, Nakazato Y, Wakabayashi K, Takahashi H, Gonatas NK 2000 Fragmentation of the Golgi apparatus of the ballooned neurons in patients 
with corticobasal degeneration and Creutzfeldt-Jakob disease. Acta Neuropathol (Berl) 100:270-274

39. Lane JD, Lucocq J, Pryde J, Barr FA, Woodman PG, Allan VJ, Lowe M 2002 Caspase-mediated cleavage of the stacking protein GRASP65 is required for Golgi fragmentation during apoptosis. J Cell Biol 156:495-509

40. Shackleton S, Hamer I, Foti M, Zumwald N, Maeder C, Carpentier JL 2002 Role of two dileucine-like motifs in insulin receptor anchoring to microvilli. J Biol Chem 277:43631-43637

41. Matter K, Mellman I 1994 Mechanisms of cell polarity: sorting and transport in epithelial cells. Curr Opin Cell Biol 6:545-554

42. Hamer I, Haft CR, Paccaud JP, Maeder C, Taylor S, Carpentier JL 1997 Dual role of a dileucine motif in insulin receptor endocytosis. J Biol Chem 272:21685-21691

43. Ghosh RN, Mallet WG, Soe TT, McGraw TE, Maxfiels FR 1998 An endocytosed TGN38 chimeric protein is delivered to the TGN after trafficking through the endocytic recycling compartment in CHO cells. J Cell Biol 142:923-936

44. van der Sluijs P, Hull M, Webster P, Male P, Goud B, Mellman I 1992 The small GTP binding protein rab4 controls an early sorting event on the endocytic pathway. Cell 70:729-740

45. Mu FT, Callagan JM, Steele-Mortimer O, Stenmark H, Parton RG, Cambell PL, McCluskey J, Yeo JP, Tock EP, Toh BH 1995 EEA1, an early endosome-associated protein. EEA1 is a conserved alpha-helical peripheral membrane protein flanked by cysteine 'fingers' and contains a calmodulin-binding IQ motif. J Biol Chem 270:13503-13511

46. Ceresa BP, Lotscher M, Schmid S 2001 Receptor and membrane recycling can occur with unaltered efficiency despite dramatic Rab5(q791)-induced changes in endosome geometry. J Biol Chem 276:9649-9654

47. Callhoun BC, Lapierre LA, Chew CS, Goldenring JR 1998 Rab11a redistributes to apical secretory canaliculus during stimulation of gastric parietal cells. Am J Physiol 275:C163-C170

48. Jedd G, Mulholland J, Segev N 1997 Two new ypt GTPases are required for exit from the yeast trans-Golgi compartment. J Cell Biol 137:563-580

49. Ullrich O, Reinsch S, UrbeS, Zerial M, Parton RG 1996 RAb11 regulates recycling through the pericentriolar recycling endosome. J Cell Biol 135:913-924
50. Urbe S, Huber LA, Zerial M, Tooze SA, Parton RG 1993 Rab11, a small GTPase associated with both constitutive and regulated secretory pathways in PC12 cells. FEBS Lett 334:175-182

51. Marks DL, Pagano RE 2002 Endocytosis and sorting of glycosphingolipids in sphingolipid storage disease. Trends Cell Biol 12:605-613

52. Abrami L, Liu S, Cosson P, Leppla SH, van der Goot FG 2003 Anthrax toxin triggers endocytosis of its receptor via a lipid raft-mediated clathrin-dependent process. J Cell Biol 160:321-328

53. Badizadegan K, Wolf AA, Rodoghiero C, Jobling M, Hirst TR, Holmes RK, Lencer WI 2002 Floating Cholera toxin into epithelial cells: functional assocoation with caveolaelike detergent-insoluble membrane microdoamins. Int J Microbiol 209:403-408

54. Harouse JM, Bhat S, Spitalnik SL, Laughlin M, Stefano K, Silberberg DH, GonzalezScarano F 1999 Inhibition of entry of HIV-1 in neural cell lines by antibodies against galactosylceramide. Science 253:320-323

55. Yahi N, Baghdiguian S, Moreau H, Fantini J 1992 Galctosylceramide (or a closely related molecule) is the receptor for the human immunodeficiency virus type 1 on human colon epithelial HT29 cells. J Virol 66:4848-4854

56. Klein TR, Kirsch D, Kaufmann R, Riesner D 2003 Prion rods contain small amounts of two host sphingolipids as revealed by thin-layer chromatography and mass spectrometry. J Biol Chem 379:655-666

57. Golde TE, Eckman CB 2001 Cholesterol modulation as an emerging strategy for the treatment of Alzheimer's disease. Drug Discov Today 6:1049-1055

58. Merrill Jr AH 2002 De novo sphingolipid biosynthesis: A necessary but dangerous pathway. J Biol Chem 277:25843-25846

59. Holthuis J, Pomorski T, Raggers RJ, Sprong H, van Meer G 2001 The organizing potential of sphingolipids in intrcellular membrane transport. Physiol Rev 31:16891723

60. Lannert H, Gorgas K, Meissner I, Wieland FT, Jeckel D 1998 Functional organization of the Golgi apparatus in glycosphingolipid biosynthesis. Lactosylceramide and subsequent glycosphingolipids are formed in the lumen of the late Golgi. J Biol Chem 273:2939-2946

61. van Meer G 1989 Lipid traffic in animal cells. Annu Rev Cell Biol 5:247-275

62. van Meer G, Lisman Q 2002 Sphingolipid transport: rafts and translocators. J Biol Chem 277:25855-25858 